Pontifícia Universidade $C_{\text {atólica }}$

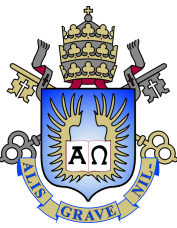

Erick Manuel Delgado Moya

Mathematical Models for the Zika Epidemic

Dissertação de Mestrado

Dissertation presented to the Programa de Pós-graduação em Matemática of PUC-Rio in partial fulfillment of the requirements for the degree of Mestre em Matemática.

Advisor: Prof. Ricardo José Alonso Plata 


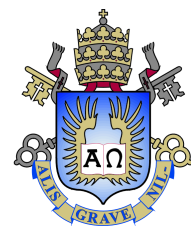

Erick Manuel Delgado Moya

\section{Mathematical Models for the Zika Epidemic}

Dissertation presented to the Programa de Pós-graduação em Matemática of PUC-Rio in partial fulfillment of the requirements for the degree of Mestre em Matemática. Approved by the undersigned Examination Committee.

Prof. Ricardo José Alonso Plata

Advisor

Departamento de Matemática - PUC-Rio

Prof. Sinésio Pesco

Departamento de Matemática - PUC-Rio

Prof. Carlos Hugo Jiménez Gómez

Departamento de Matemática - PUC-Rio

Prof. Paulo Verdasca Amorim

Universidade Federal do Rio de Janeiro - UFRJ

Prof. Márcio da Silveira Carvalho

Vice Dean of Graduate Studies

Centro Técnico Científico - PUC-Rio 
All rights reserved.

\section{Erick Manuel Delgado Moya}

Has a degree in mathematics and master degree in mathematics with specialization in Optimization from University of Havana. I work in the area of Biomathematics specialized in Epidemiology.

Bibliographic data

Delgado Moya, Erick Manuel

Mathematical Models for the Zika Epidemic / Erick Manuel Delgado Moya; advisor: Ricardo José Alonso Plata. - Rio de janeiro: PUC-Rio, Departamento de Matemática, 2018.

v., 65 f: il. color. ; $30 \mathrm{~cm}$

Dissertação (Mestrado) - Pontifícia Universidade Católica do Rio de Janeiro, Departamento de Matemática.

Inclui bibliografia

1. Matemática - Dissertação. 2. Controle;. 3. Equações diferencias ordinárias;. 4. Modelos;. 5. Retardo;. 6. Transmissão;. 7. ZIKV.. I. Alonso Plata, Ricardo Jose. II. Pontifícia Universidade Católica do Rio de Janeiro. Departamento de Matemática. III. Título. 

and father. 


\section{Acknowledgments}

I dedicate this work to my mother and my sister who always support me and who are with me at all times.

To my friends and colleagues Dania, Orlando, Yunelsy and Raimundo for their good advice and willingness to help me.

I would like to express my sincere gratitude to Aymee for her friendship and the unconditional love and for trusting me.

To Maria Esther, Pedro and Odalys for her eternal friendship and being the person who understands me and does not judge me.

I would like to express my sincere gratitude to Elina Miret and Juan Manuel Otero for their great support and confidence.

To my advisor Ricardo Alonso for giving me the opportunity to work with him and the freedom to work on this theme.

I would like to express my sincere gratitude to Claudia and Adrian for the good conversations and their unconditional help.

My sincere thanks also goes to Creuza, Katia and Carlos (administrative staff of the Department of Mathematics) for helping me to fulfill all the bureaucracy requirements and for your friendship.

I would like to express my sincere gratitude to CAPES for the aid granted. 


\section{Abstract}

Delgado Moya, Erick Manuel; Alonso Plata, Ricardo Jose (Advisor). Mathematical Models for the Zika Epidemic. Rio de Janeiro, 2018. 65p. Dissertação de Mestrado - Departamento de Matemática, Pontifícia Universidade Católica do Rio de Janeiro.

The Zika Virus (ZIKV) is a virus transmitted by Aedes aegypti mosquitoes (same as the one transmitting dengue and chikungunya fever) and Aedes albopictus. The main way of contagion by the ZIKV is caused by the bite of a mosquito that, after feeding from someone contaminated, can transport the ZIKV throughout its life, transmitting the disease to a population that does not have the immunity. It can also be transmitted through a person's sexual relationship with ZIKV to their partners, even if the infected person does not have the symptoms of the disease. In this work, we present two mathematical models for the ZIKV epidemic by using (1) ordinary differential equations and, (2) ordinary differential equations with temporal delay, which is the time it takes mosquitoes to develop the virus. We make a comparison between the two modeling variants and, to facilitate the work with the models, we provide a graphical user interface. Computational simulations are performed for Suriname and El Salvador, which are countries that are prone to develop the epidemic in an endemic manner. In order to study the spatial diffusion of ZIKV, we propose a model based on advection-diffusion equations and create a numerical scheme with finite elements and finite differences to resolve it.

\section{Keywords}

Control; Ordinary differential equations; Models; Delay; Transmission; ZIKV. 


\section{Resumo}

Delgado Moya, Erick Manuel; Alonso Plata, Ricardo Jose. Modelos Matemáticos para a Epidemia do Zika. Rio de Janeiro, 2018. 65p. Dissertação de Mestrado - Departamento de Matemática, Pontifícia Universidade Católica do Rio de Janeiro.

Zika Vírus (ZIKV) é um vírus transmitido pelos mosquitos Aedes aegypti (mesmo transmissor da dengue e da febre chikungunya) e o Aedes albopictus. O contágio principal pelo ZIKV se dá pela picada do mosquito que, após se alimentar do sangue de alguém contaminado, pode transportar o ZIKV durante toda a sua vida, transmitindo a doença para uma população que não possui anticorpos contra ele. Também pode ser transmitido através de relação sexual de uma pessoa com Zika para os seus parceiros ou parceiras, mesmo que a pessoa infectada não apresente os sintomas da doença.Neste trabalho, apresentamos dois modelos matemáticos para a epidemia do ZIKV usando (1) equações diferenciais ordinárias e (2) equações diferenciais ordinárias com atraso temporal, que é o tempo que os mosquitos levam para desenvolver o vírus. Fazemos uma comparação entre as duas variantes de modelagem e, para facilitar o trabalho com os modelos, fornecemos uma interface gráfica com o usuário. Simulações computacionais são realizadas para o Suriname e El Salvador, que são países propensos a desenvolver a epidemia de maneira endêmica. Para estudar a difusão espacial do ZIKV, propomos um modelo baseado em equações de advecção-difusão e criamos um esquema numérico com elementos finitos e diferenças finitas para resolvê-lo.

\section{Palavras-chave}

Controle; Equações diferencias ordinárias; Modelos; Retardo; Transmissão; ZIKV. 


\section{Table of contents}

1 Introduction $\quad 13$

$\begin{array}{lll}1.1 & \text { Preliminars ZIKV } & 13\end{array}$

1.2 Dissertation Outline 14

2 Background $\quad 15$

2.1 Differential Ordinary Equation $\quad 15$

$\begin{array}{ll}2.2 \text { Differential Equation with Delay } & 16\end{array}$

$\begin{array}{ll}2.3 \text { Basic Reproduction Number }\left(\Re_{0}\right) & 19\end{array}$

3 Mathematical Model for ZIKV Transmission $\quad 24$

3.1 Model with Exposed Variable 24

3.2 Model with Temporal Delay 34

3.3 Numerical Simulations 37

4 Preliminary work on diffusive-advective model for spatial evolution of ZIKV epidemic

4.1 Background 42

4.2 Advection-Diffusion Model for spatial evolution of ZIKV epidemic 49

4.3 Method of solution using FEM / Crank-Nicolson 52

$5 \quad$ Graphical User Interface $\quad 57$

5.1 Graphical User Interface $\quad 57$

6 Appendix $\quad 60$

Bibliography $\quad 62$ 


\section{List of figures}

Figure 3.1.1 Contagion by mosquitoes. 25

Figure 3.1.2 Sexual contagion. 26

Figure 3.1.3 Schematic Representation. 26

Figure 3.2.1 Schematic Representation of Model with Temporal De-

lay. $\quad 34$

Figure 3.3.1 $\Re_{0}^{m} \quad 39$

Figure 3.3.2 Comparison between models. Suriname Infected. 40

Figure 3.3.3 First Moment of Coincidence. Suriname infected. 40

Figure 3.3.4 Infected humans at 360 days. Suriname. 40

Figure 3.3.5 Comparison between models. El Salvador Infected. 41

Figure 3.3.6 First Moment of Coincidence. El Salvador infected. 41

Figure 3.3.7 Infected humans at 360 days. El Salvador. 41

Figure 4.2.1 Schematic representation of the diffusion model. $\quad 51$

Figure 5.1.1 Graphical User Interface. $\quad 57$

Figure 5.1.2 Graphical User Interface, button Help. 58

Figure 5.1.3 Graphical User Interface, button Infected Humans. 58

Figure 5.1.4 Graphical User Interface, button Comparison. 59

Figure 5.1.5 Graphical User Interface, button Mosquitoes. 59 


\section{List of tables}

Table 3.1 Description of parameters used in the model. 25

Table 3.2 Suriname, $2016 . \quad 38$

Table 3.3 El Salvador, $2016 . \quad 38$

Table 3.4 Initial Conditions, 2016. 38

Table 4.1 Description of parameters used in the model 4.20. 50 


\section{List of Abreviations}

ODEs - Ordinary Differential Equations

PDEs - Partial Differential Equations

DDEs - Delay Differential Equations

FEM - Finite Elements Method

ZIKV - Zika virus 


\section{Introduction}

\section{1}

\section{Preliminars ZIKV}

Zika fever (also known as Zika virus disease) is an illness caused by the Zika virus. The disease is spread through the bite of daytime-active Aedes mosquitoes such as the A. aegypti and A. albopictus (these are the same mosquitoes that spread dengue and chikungunya viruses). Its name comes from Zika forest in Uganda, where the virus was first isolated from a rhesus monkey in 1947. The first human cases were reported in Nigeria in 1954. The first documented outbreak among people occurred in 2007, in the Federated State of Micronesia [28].

The disease of Zika virus is transmitted from infected Aedes mosquitoes to humans through mosquito bites [16]. It can also be transmitted from human to human through the blood and semen of an infected human, and through an infected pregnant woman to the foetus. Zika is a cause of microcephaly and other severe brain defects [6]. The incubation period (the time from exposure to symptoms) of Zika virus disease is not clear, but is likely to be a few days to a week. The symptoms are similar to other arbovirus infection such as dengue, and include fever, skin rashes, conjunctivitis (red eyes), muscle and joint pain, malaise and headache. These symptoms are usually mild and usually last from 2- 7 days [16].

There is no specific treatment or vaccine currently available for Zika virus disease. Prevention and control relies on reducing mosquitoes through source reduction (removal and modification of breeding sites), and reducing contacts between mosquitoes and people.

The use of ODE (ordinary differential equation) and ODE with delay in the study of epidemics can be seen in [2, 21], for Dengue in [19, 35], for HIV/AIDS in [1, 39], for Ebola in $[4,13]$ and ZIKA in $[6,28,33]$. The use of advection-diffusion equations in the study of epidemics can be found in $[22,29]$, in particular for Dengue $[23,26,36]$, for HIV / AIDS in [20, 31] and for Malaria in [25] these texts contributed to the background of this work.

The objective of this work is to present mathematical models for the 
transmission and diffusion of the ZIKV, using classical ordinary differential equations, with discrete time delay and advesion-diffusion equations, these models take into account sexual transmission and are stratified by sex and help take into account most of the epidemic's contagion.

\section{2}

\section{Dissertation Outline}

This dissertation is organized in six chapters. Chapter 1 presents the general information regarding the ZIKV. Chapter 2 contains the definitions, theorems and methods development of the dissertation.

In Chapter 3 we present two mathematical models for ZIKV and perform computational experiments for Suriname and El Salvador.

Chapter 4 contains a model to study the diffusion of the ZIKV and a numerical scheme is proposed to resolve it. And as a future work, we have the study of the model and computational experimentation.

Chapter 5 has a graphical user interface that allows to work with the model more easily and quickly.

Chapter 6 contains the enunciate and demonstration de lemmas use in the dissertation. 


\section{2}

\section{Background}

In this chapter, we present the basic theory of ordinary differential equation [7, 8, 27], ordinary differential equation with delay [3, 18], finite element method [9] and Crank-Nicolson method [10] of finite difference, used in the development of work.

We define the basic reproductive number $\left(\Re_{0}\right)$ and its relation to local stability at the disease-free equilibrium $[14,17]$.

\section{1}

\section{Differential Ordinary Equation}

\section{Existence and Uniqueness of Solution}

Theorem 2.1. (Existence and Uniqueness Theorem)

Let:

$$
\begin{gathered}
x_{1}^{\prime}=f_{1}\left(x_{1}, x_{2}, \ldots, x_{n}, t\right), x_{1}\left(t_{0}\right)=x_{10} \\
x_{2}^{\prime}=f_{2}\left(x_{1}, x_{2}, \ldots, x_{n}, t\right), x_{2}\left(t_{0}\right)=x_{20} \\
\vdots \\
x_{n}^{\prime}=f_{n}\left(x_{1}, x_{2}, \ldots, x_{n}, t\right), x_{n}\left(t_{0}\right)=x_{n 0} .
\end{gathered}
$$

Suppose $D$ is the region in $(n+1)$-dimensional space (one dimension for $t$ and $n$ dimension for the vector $x)$. If the partial derivative $\frac{\partial f_{i}}{\partial x_{j}}, i, j=1,2, \ldots, n$ are continuous in

$$
D=\left\{(x, t):\left|t-t_{0}\right| \leq a,\left|x-x_{0}\right| \leq b\right\}
$$

then there is a constant $\delta>0$ such that exists a unique continuous vector solution $x=\left[x_{1}(t), x_{2}(t), \ldots, x_{n}(t)\right]$ in the interval $\left|t-t_{0}\right| \leq \delta$.

The enunciate and the proof of this theorem is in [13]. 


\section{2}

\section{Differential Equation with Delay}

Delay differential equations (DDEs) are a type of differential equation in which the derivative of the unknown function at a certain time is given in terms of the values of the function at previous times. DDEs are also called time-delay systems, systems with after effect or dead-time, hereditary systems, equations with deviating argument, or differential-difference equations. They belong to the class of systems with the functional state, partial differential equations (PDEs) which are infinite dimensional, as opposed to ordinary differential equations (ODEs) having a finite dimensional state vector.

\section{Existence of Solution}

Consider the nonlinear delay differential equation

$$
x^{\prime}(t)=f(t, x(t), x(t-r))
$$

with a single delay $r>0$. Assume that $f(t, x, y)$ and $f_{x}(t, x, y)$ are continuous on $\mathbb{R}^{3}$. Let $s \in \mathbb{R}$ be given and let $\phi:[s-r, s] \rightarrow \mathbb{R}$ be continuous. We seek a solution $x(t)$ of $(2.2)$ satisfying

$$
x(t)=\phi(t), s-r \leq t \leq s
$$

and satisfying (2.2) on $s \leq t<s+p$ for some $p>0$. Note that we must interpret $x^{\prime}(s)$ as the right-hand derivative at $s$.

The equation (2.2) can be solved by the method of steps as follows. For $s \leq t \leq s+r, x(t)$ must satisfy the initial-value problem for the ODE:

$$
y^{\prime}(t)=f(t, y(t), \phi(t-r), y(s)), y(s)=\phi(s), \quad s \leq t \leq s+r .
$$

As $e(t, y)=f(t, y(t), \phi(t-r))$ and $e_{y}(t, y)$ (derivative respect to $y$ ) are continuous, a local solution of this ODE is guaranteed by standard results from ODE theory. If this local solution $x(t)$ exists for the entire interval $s \leq t \leq s+r$, then our solution $x(t)$ is defined so far on $[s-r, s+r]$ and we may repeat the above argument to extend our solution still father to the right. Indeed, for $s+r \leq t \leq s+2 r$, a solution $x(t)$ of (2.2)-(2.3) must satisfy the initial valueproblem for the ODE:

$$
y^{\prime}(t)=f(t, y(t), \phi(t-r), y(s+r))=x(s+r), \quad s+r \leq t \leq s+2 r .
$$

Again, standard existence results for such problems guarantee the existence of a unique solution, which we call $x(t)$, defined on some subinterval 
$[s+r, p) \subset[s+r, s+2 r]$, possibly the entire interval. Of course, $x(t)$, now defined on $[s-r, p)$ where $p>s+r$, is a solution of (2.2)-(2.3). If the solution exists on the entire interval $[s+r, s+2 r]$ then we may again repeat the process to extend the solution to $[s+2 r, s+3 r]$, or some subinterval of this interval.

Theorem 2.2. Let $f(t, x, y)$ and $f_{x}(t, x, y)$ be continuous on $\mathbb{R}^{n}, s \in \mathbb{R}$, and let $\phi:[s-r, s] \rightarrow \mathbb{R}$ be continuous. Then there exists $p>s$ and a unique solution of the initial-value problem (2.2)-(2.3) on $[s-r, p]$.

The enunciate and proof is in [18].

The Theorem 2.2 provides only a local solution. Just as for ODEs, we can often, but not always, extend this solution to be defined for all $t \geq s$.

The next result shows that if a noncontinuable solution is not defined for all $t \geq s-r$, then it must blow up as $t \rightarrow p$. This is the same conclusion as for the ODE theory; in fact that theory proves the result.

Theorem 2.3. Let $f$ satisfy the hypotheses of Theorem 2.2 and let $x$ : $[s-r, p) \rightarrow \mathbb{R}$ be the noncontinuable solution of the initial- value problem (2.2)-(2.3). If $p<\infty$ then

$$
\lim _{t \rightarrow p^{-}}|x(t)|=\infty
$$

Proof. Let $x:[s-r, p) \rightarrow \mathbb{R}$ be the noncontinuable solution and suppose that $p<\infty$. Then $s+j r<p \leq s+(j+1) r$ for some $j \in\{0,1,2, \ldots$,$\} so the$ restriction of $x(t)$ to the interval $[s+r j, p)$ is necessarily the noncontinuable solution of the initial-value problem for the ODE:

$$
y^{\prime}=f(t, y(t), x(t-r), y(s+j r))=x(s+j r)
$$

inasmuch as any extension of it would give an extension of $x(t)$ as a solution of (2.2)- (2.3). But then the result follows from the continuation theorem for ODEs.

\section{Positivity of Solution}

Theorem 2.4. Suppose that $f: \mathbb{R} \times \mathbb{R}_{+}^{n} \times \mathbb{R}_{+}^{n} \rightarrow \mathbb{R}^{n}$ satisfies the hypotheses of Theorem 2.2 and

$$
\forall i, t, \forall x, y \in R_{+}^{n}: x_{i}=0 \Rightarrow f_{i}(t, x, y) \geq 0
$$

If the initial data $\phi$ in (2.3) satisfy $\phi \geq 0$, then the corresponding solution $x(t)$ of (2.2) satisfies $x(t) \geq 0$ for all $t \geq s$ where it is defined, see [18]. 
Proof. Recall that the analogous result for ODEs $x^{\prime}=g(t, x)$ where $g$ : $\mathbb{R} \times \mathbb{R}_{+}^{n} \rightarrow \mathbb{R}^{n}$ requires $g$ to satisfy $g_{i}(t, x) \geq 0$ whenever $x \in \mathbb{R}_{+}^{n}$ satisfies $x_{i}=0$.

Let $x(t)$ denote the solution of (2.2)-(2.3). On the interval $s \leq t \leq s+r, x(t)$ satisfies the ODE $x^{\prime}(t)=g(t, x(t))$ where $g(t, x)=f(t, x, \phi(t-r)) . g$ is easily seen to satisfy the conditions in the previous paragraph and hence $x(t) \geq 0$ on the interval $s \leq t \leq s+r$. Now one just repeats the argument using the method of steps.

Proposition 2.1. If $x:[p-r, p+a] \rightarrow \mathbb{R}^{n}$ is continuous, then $t \rightarrow x_{t}$ is a continuous function from $[p, p+a]$ into $C\left([-r, 0], \mathbb{R}^{n}\right)$, refer to [18].

Proof. As $x$ is uniformly continuous on the closed, bounded interval $I=$ $[p-r, p+a]$, given $\epsilon>0, \exists \delta>0$, such that $t, s \in I,|t-s|<\delta$ $\Rightarrow|x(t)-x(s)|<\epsilon$. This implies that

$$
\left|x_{t}(\sigma)-x_{s}(\sigma)\right|=|x(t+\sigma)-x(s+\sigma)|<\epsilon
$$

for $\sigma \leq t, s \leq \sigma+a$ with $|t-s|<\delta$ and $-r \leq \sigma \leq 0$, proving the result.

\section{Small Delays are Harmless}

Consider the linear delay system

$$
x^{\prime}(t)=A x(t)-B x(t-r)
$$

and its nondelayed counterpart obtained by setting $r=0$,

$$
x^{\prime}(t)=(A+B) x(t) .
$$

We want to explore the correspondence of the characteristic roots of

$$
h(\lambda, r)=\operatorname{det}[\lambda I-A-\exp (-\lambda r) B]=0
$$

to the eigenvalues of $A+B$ :

$$
h(\lambda, 0)=\operatorname{det}[\lambda I-(A+B)]=0 .
$$

Proposition 2.2. Given $\sigma \in \mathbb{R}$, there are at most finitely many characteristic roots satisfying $\Re(\lambda)>\omega$. If there are infinitely many distinct characteristic roots $\left\{\lambda_{n}\right\}_{n}[18]$, then

$$
R\left(\lambda_{n}\right) \rightarrow-\infty, n \rightarrow \infty
$$


Theorem 2.5. Let $z_{1}, z_{2}, \ldots, z_{k}$ be the distinct eigenvalues of $A+B$ let $\delta>0$, and let $s \in \mathbb{R}$ satisfy $s<\min _{i} \Re\left(z_{i}\right)$. Then there exists $r_{0}>0$ such that if $0<r<r_{0}$ and $h(z, r)=0$ for some $z$ then either $\Re(z)<s$ or $\left|z-z_{i}\right|<\delta$ for some $i$, see [18].

Proof. Let $G=\left\{z \in \mathbb{C}: \Re(z) \geq s,\left|z-z_{i}\right| \geq \delta, 1 \leq i \leq k\right\}$. Our goal is to show that there are no characteristic roots in the closed set $G$ if $r$ is small enough. If this were false, then there exists a sequence $\left\{r_{n}\right\}_{n}$ of delay with $r_{n}>0$ and $r_{n} \rightarrow 0$ and a corresponding sequence of characteristic roots $z_{n} \in G$ so that $h\left(z_{n}, r_{n}\right)=0$. By the Bolzano-Weierstrass theorem there are two cases:

1. $\left\{z_{n}\right\}$ has a convergent subsequence converging to $z^{*} \in G$ ( $G$ is closed).

2. $\left|z_{n}\right| \rightarrow \infty$.

In case 1 , continuity of $h$ implies that $h\left(z^{*}, 0\right)=0$ but $A+B$ has no eigenvalues in $G$. This case cannot occur. We conclude that case 2. must hold. Now we argue exactly as in the proof of Proposition (2.2) that $C_{n}=$ $I-\frac{1}{z_{n}}\left[A+\exp \left(-z_{n} r_{n}\right) B\right] \rightarrow I$ because $\Re\left(z_{n}\right) \geq$ s and $\left|z_{n}\right| \rightarrow \infty$ so it cannot be singular for larger $n$. This contradiction proves the result.

Small delays are harmless in the sense that if asymptotic stability holds when $\tau=0$, then it continues to hold for small delays inasmuch as we may choose $\delta$ small enough that $\delta$ - ball about each eigenvalues of $A+B$ belongs to the left half- plane and we may choose $s$ negative. On the other hand, if instability holds for $\tau=0$ due to a simple positive root or a complex conjugate pair of roots with positive real part, then the implicit function theorem may be applied to show that instability continues to hold for small $r>0$.

\section{3 \\ Basic Reproduction Number $\left(\Re_{0}\right)$}

The basic reproduction number $\Re_{0}$ is arguably the most important quantity in infectious disease epidemiology. It is among the qualities most urgently estimated for emerging infectious diseases in outbreak situations, and its value provides insight when designing control interventions for established infections. From a theoretical point of view $\Re_{0}$ plays a vital role in the analysis of, and consequent insight from, infectious disease models. $\Re_{0}$ is defined as the average number of an infection caused by one typical infected individual, in a population consisting of susceptible only [17].

It has been shown that $\Re_{0}$ is mathematically characterized by regarding infection transmission as a demographic process, where producing offspring is not seen as giving birth in the demographic sense, but as causing a new 
infection through transmission. In a natural way, this leads to viewing the infection process in terms of consecutive generations of infected individuals, in complete analogy to demographic generations. Subsequent generations growing in size then indicate a growing population, and the growth factor per generation indicates the potential for growth. In a natural way, this growth factor is then the mathematical characterization of $\Re_{0}$.

If $0<\Re_{0}<1$ the infection will die out in the long run. But if $\Re_{0}>1$ the infection will be able to spread in a population [17].

Generally, the larger the value of $\Re_{0}$, the harder it is to control the epidemic. The basic reproduction number is affected by several factors including the duration of infectivity of affected patients, the infectiousness of the organism, and the degree of contact between the susceptible and affected populations. To calculate $\Re_{0}$ one begins with those equations of the ODE system that describe the production of new infections and changes in state among infected individuals. We will refer to the set of such equations as the infected subsystem. The first step is to linearize the infected subsystem of nonlinear ODEs about the infection-free steady state that, as a rule, exists. Epidemiologically, the linearization reflects that $\Re_{0}$ characterizes the potential for initial spread of an infectious agent when it is introduced into a fully susceptible population and that we assume that the change in the susceptible population is negligible during the initial spread. This linearized infected subsystem is the starting point of our calculations.

\section{The relationship between $\Re_{0}$ and local stability in disease-free point}

The basic reproduction number cannot be determined from the structure of the mathematical model alone, but depends on the definition of infected and uninfected compartments. We define $X_{s}$ to be the set of all disease free states. That is

$$
X_{s}=\left\{x \geq 0 \mid x_{i}=0, i=1, \ldots, m\right\} .
$$

Let $T_{i}(x)$ be the rate of appearance of new infections in compartment $i$, $\Sigma_{i}^{+}(x)$ be the rate of transfer of individuals into compartment $i$ by all other means, and $\Sigma_{i}^{-}(x)$ be the rate of transfer of individuals out of compartment $i$. It is assumed that each function is continuously differentiable at least twice in each variable. The disease transmission model consists of non- negative initial conditions together with the following system of equations:

$$
x^{\prime}=T_{i}(x)-\Sigma_{i}(x)=f_{i}(x), \quad i=1, \ldots, n,
$$

where $\Sigma_{i}=\Sigma_{i}^{-}-\Sigma_{i}^{+}$and the functions satisfy assumptions described below: 
(P1) Since each function represents a directed transfer of individuals, they are all non-negative.

If $x \geq 0$, then $T_{i}, \Sigma_{i}^{-}, \Sigma_{i}^{+} \geq 0$ for $i=1, \ldots, m$.

(P2) If a compartment is empty, then there can be no transfer of individuals out of the compartment by death, infection, nor any other means. Thus, if $x_{0}=0$ then $\Sigma_{i}^{-}(x)=0$.

(P3) The incidence of infection for uninfected compartments is zero. $T_{i}=0$ if $i>m$.

(P4) There is no (density independent) immigration of infectives. This condition is stated as follows:

If $x \in X_{s}$ then $T_{i}(x)=0$ and $\Sigma_{i}^{+}(x)=0$ for $i=1, \ldots, m$.

(P5) Consider a population near the equilibrium disease-free point $x_{0}$. If the population remains near the $x_{0}$ ( if the introduction of a few infective individuals does not result in an epidemic) then the population will return to the $x_{0}$ according to the linearized system

$$
x^{\prime}=\operatorname{Df}\left(x_{0}\right)\left(x-x_{0}\right)
$$

where $D f\left(x_{0}\right)$ is the derivative $\frac{\partial f_{i}}{\partial x_{j}}$ evaluated at the $x_{0}$ (the Jacobian matrix in $x_{0}$ ). Here, and in what follows, some derivatives are one sided, since $x_{0}$ is on the domain boundary. We restrict our attention to systems in which the $x_{0}$ is stable in the absence of new infection. That is, If $T(x)$ is set to zero, then all eigenvalues of $D f\left(x_{0}\right)$ have negative real parts.

The conditions listed above allow us to partition the matrix $D f\left(x_{0}\right)$ as shown by the following lemma.

Lemma 2.1. If $x_{0}$ is a the equilibrium disease-free point of system (2.5) and $f_{i}(x)$ satisfies $(P 1)$ - (P5), then derivatives $D T\left(x_{0}\right)$ and $D \Sigma\left(x_{0}\right)$ are partitioned as

$$
\begin{gathered}
D T\left(x_{0}\right)\left[\begin{array}{ll}
T & 0 \\
0 & 0
\end{array}\right] \\
D \Sigma\left(x_{0}\right)\left[\begin{array}{ll}
\Sigma & 0 \\
J_{3} & J_{4}
\end{array}\right]
\end{gathered}
$$


where $T$ and $\Sigma$ are the $m \times m$ matrices defined by $T=\left[\frac{\partial T_{i}}{\partial x_{j}}\right], \Sigma=\left[\frac{\partial \Sigma_{i}}{\partial x_{j}}\right]$ with $1 \leq i, j \leq m$. Further, $T$ is non-negative, $\Sigma$ is a non-singular M-matrix ${ }^{1}$ and all eigenvalues of $J_{4}$ have positive real part.

The enunciate and proof is in [14].

To interpret the entries of $T \Sigma^{-1}$ and develop a meaningful definition of $\Re_{0}$, consider the fate of an infected individual introduced into compartment $k$ of a disease-free population. The $(j, k)$ entry of $\Sigma^{-1}$ is the average length of time this individual spends in compartment $j$ during its lifetime, assuming that the population remains near the equilibrium disease-free and barring reinfection. The $(i, j)$ entry of $T$ is the rate at which infected individuals in compartment $j$ produce new infections in compartment $i$. Hence, the $(i, k)$ entry of the product $T \Sigma^{-1}$ is the expected number of new infections in compartment $i$ produced by the infected individual originally introduced into compartment $k$. We call $T \Sigma^{-1}$ the next generation matrix for the model and set

$$
\Re_{0}=\rho\left(-T \Sigma^{-1}\right)
$$

where $\rho(A)$ denotes the spectral radius of a matrix $A$.

The following theorem shows the relationship between the $\Re_{0}$ and the local stability in $x_{0}$.

Theorem 2.6. Consider the disease transmission model given by (2.5) and $f_{i}(x)$ satisfies (P1)- (P5). If $x_{0}$ is the equilibrium disease-free point of the model, the $x_{0}$ is locally asymptotically stable if $\Re_{0}<1$, but unstable if $\Re_{0}>1$ with $\Re_{0}=\rho\left(-T \Sigma^{-1}\right)$.

Proof. Let $J_{1}=T-\Sigma$. Since $\Sigma$ is non-singular $M$-matrix and $T$ is nonnegative, $-J_{1}=\Sigma-T$, has the $Z$ sign pattern ${ }^{2}$. Thus,

$$
s\left(J_{1}\right)<0 \Leftrightarrow-J_{1} \text { is a non- singular matrix, }
$$

where $s\left(J_{1}\right)$ denotes the maximum real part of all the eigenvalues of the matrix. Since $T \Sigma^{-1}$ is non-negative $-J_{1} \Sigma^{-1}=I-T \Sigma^{-1}$ also has the $Z$ sign pattern. For the Lemma 6.2 with $H=\Sigma$ and $B=-J_{1}=\Sigma-T$,

$-J_{1}$ is a non-singular $M$-matrix $\Longleftrightarrow I-T \Sigma^{-1}$ is a non-singular $M$-matrix.

${ }^{1}$ If $\mathrm{B}=$ sI-P, where $I$ is the identity matrix, $P$ is non-negative, and $s>\rho(P)(s(A)$ be the maximum real part of the eigenvalues of $A$ (the spectral abscissa), and let $\rho(A)$ be the maximum modulus of the eigenvalues of A (the spectral radius)), then B is non-singular Mmatrix, if $s=\rho(P)$, then $B$ is a singular M-matrix.

${ }^{2} \mathrm{~A}$ matrix $B=\left[b_{i j}\right]$ has the sign pattern if $b_{i j} \leq 0$ for all $i \neq j$. 
Finally, since $T \Sigma^{-1}$ is non- negative, all eigenvalues of $T \Sigma^{-1}$ have magnitude less than or equal to $\rho\left(T \Sigma^{-1}\right)$. Thus,

$$
I-T \Sigma^{-1} \text { is a non- singular M-matrix, } \Longleftrightarrow \rho\left(T \Sigma^{-1}\right)<1 \text {. }
$$

Hence,

$$
s\left(J_{1}\right)<0 \text { if and only if } R_{0}<1 \text {. }
$$

Similarly, it follows that

$$
\begin{aligned}
s\left(J_{1}\right)=0 & \Longleftrightarrow-J_{1} \text { is a singular M-matrix }, \\
& \Longleftrightarrow I-T \Sigma^{1} \text { is a singular M-matrix } \\
& \Longleftrightarrow \rho\left(T \Sigma^{-1}\right)=1
\end{aligned}
$$

The second equivalence follows from Lemma 6.3 of Appendix, with $H=\Sigma$ and $K=T$. The remainder of the equivalences follows as with the non-singular case. Hence, $s\left(J_{1}\right)=0$ if and only if $\Re_{0}=1$. It follows that $s\left(J_{1}\right)>0$ if and only if $\Re_{0}>1$. 


\section{Mathematical Model for ZIKV Transmission}

The use of ODE (ordinary differential equation) and ODE with delay in the study of epidemics can be seen in $[2,21]$, in particular for Dengue in [19, 35], for HIV/AIDS in [1, 39], for Ebola in [4, 13] and ZIKA in [6, 28, 33], these texts contributed as background in the work that we present.

The objective of this work is to present models for the ZIKV epidemic based on ODE and ODE with delay. A theoretical study of the model was made and the $\Re_{0}$ was calculated for the sub-model with only contagion by mosquitoes and with only sexual contagion. Computational simulations are performed in Suriname and El Salvador, where ZIKV can become endemic. We performed a comparison between the two variants of modeling with respect to the time of the epidemic and the number of infected.

\section{1}

\section{Model with Exposed Variable}

The model variables are susceptible men $H_{s}$, susceptible women $M_{s}$, exposed men $H_{E}$, exposed women $M_{E}$, infected men $H_{I}$, infected women $M_{I}$, recovered men $H_{R}$, recovered women $M_{R}$, susceptible mosquitoes $V_{s}$ and infected mosquitoes $V_{I}$. The parameters of the model are between 0 and 1 and are described in Table (3.1).

Let:

$\sigma_{v}$ : Number of times a single mosquito bites a human per unit time.

$\beta_{h v}$ : Probability of pathogen transmission from an infectious mosquito to a susceptible human given that a contact between the two occurs.

$\beta_{v h}$ : Probability of pathogen transmission from an infectious human to a susceptible mosquito given that a contact between the two occurs.

$N_{h}$ : Represent the total population of human within the model and remain constant.

Then [33],

$$
\begin{aligned}
& \beta_{y_{1}}=\frac{\sigma_{v} \beta_{h v}}{N_{h}}=\frac{\beta_{y_{1 *}}}{N_{h}} \\
& \beta_{x}=\frac{\sigma_{v} \beta_{v h}}{N_{h}}=\frac{\beta_{x *}}{N_{h}}
\end{aligned}
$$


To define $\beta_{y_{2}}$ and $\beta_{y_{3}}$ we did an analogous study but taking into account the sexual contacts (between men and heterosexual respectively) and the probability of infecting these contacts.

\begin{tabular}{|l|l|}
\hline Parameters & Description \\
\hline$\beta_{y_{1}}$ & The force of infection from infected mosquito to susceptible human \\
\hline$\beta_{y_{2}}$ & The force of infection from infected man to susceptible man \\
\hline$\beta_{y_{3}}$ & The force of infection from infected man to susceptible woman \\
\hline$\beta_{x}$ & The force of infection from infected human to susceptible mosquito \\
\hline$\mu_{1}, \mu_{2}, \eta$ & Man, woman and mosquito natural death rates \\
\hline$\omega_{1}, \omega_{2}, \omega_{3}$ & $\begin{array}{l}\text { The rate of progression of men, women and mosquitoes from the exposed state to the } \\
\text { infectious state }\end{array}$ \\
\hline$\epsilon_{1}, \epsilon_{2}$ & Disease-induced death rate for humans (man and woman) \\
\hline$r_{1}, r_{2}$ & Per capital recovery rate for humans from the infectious (man and woman) \\
\hline$N_{1}$ & Entry rate of susceptible men \\
\hline$N_{2}$ & Entry rate of susceptible women \\
\hline$N_{3}$ & Entry rate of susceptible mosquitoes \\
\hline
\end{tabular}

Table 3.1: Description of parameters used in the model.

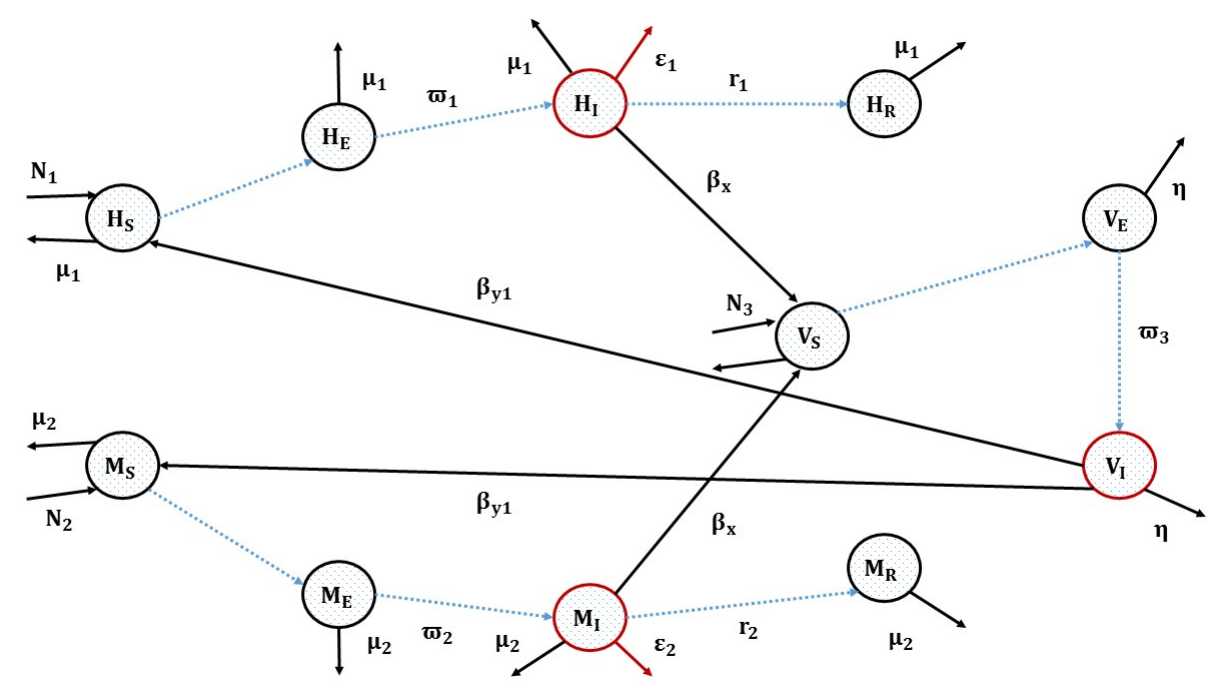

Figure 3.1.1: Contagion by mosquitoes. 


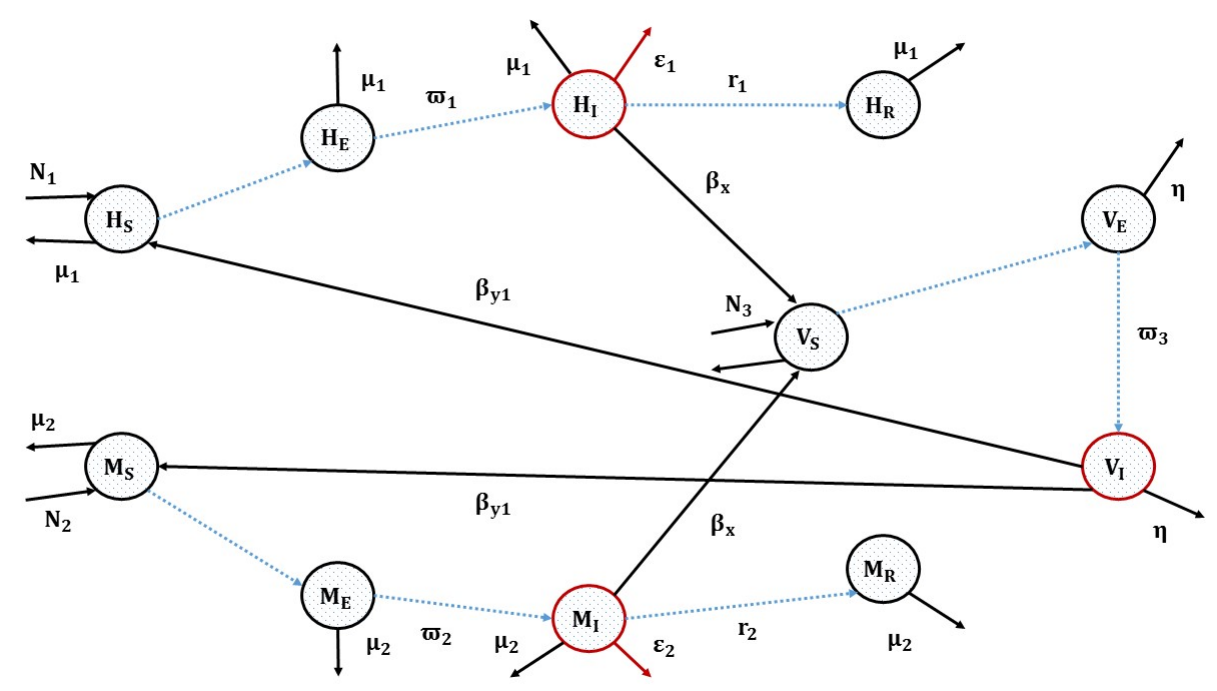

Figure 3.1.2: Sexual contagion.

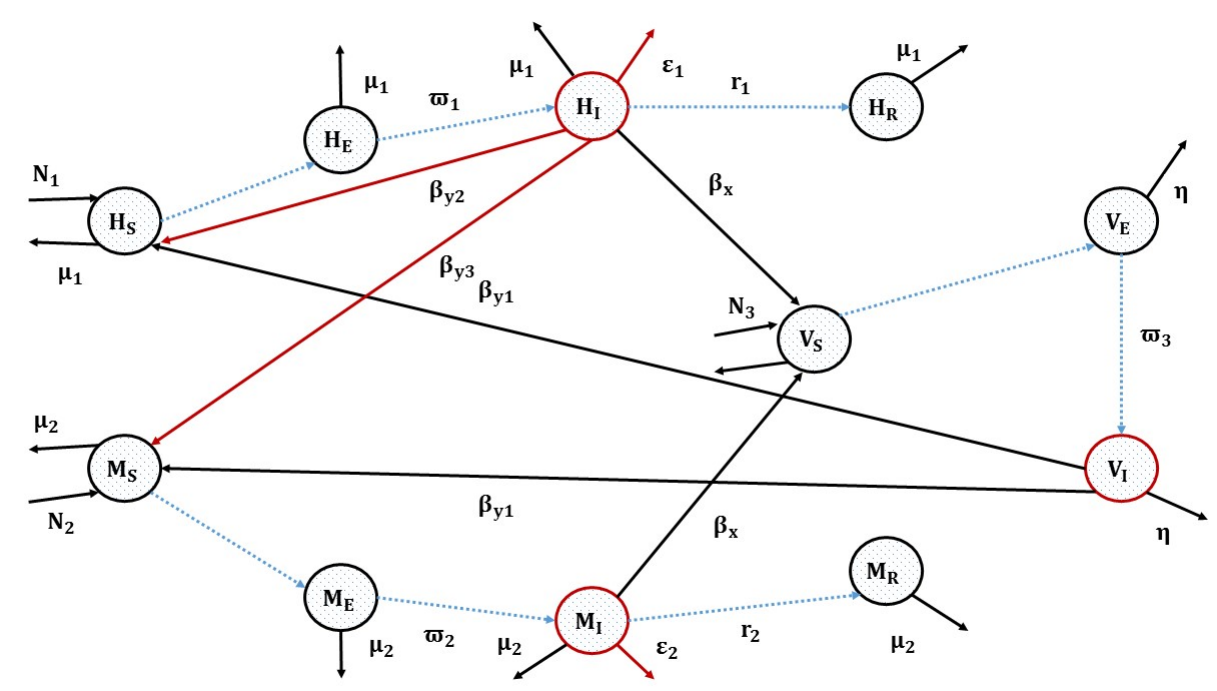

Figure 3.1.3: Schematic Representation. 
The figure (3.1.3) is A schematic representation of model (3.1), modeling the progression of ZIKV in human and mosquito populations. Susceptible humans start in $H_{s}, M_{s}$ (men and women) and move to $H_{E}$ and $M_{E}$, the exposed population, once infected by a mosquito carrying the virus and sexual contact. After an intrinsic incubation period, exposed individuals become infectious population and moves to the infectious population, $H_{I}$ and $M_{I}$. Infectious humans will then move to and remain in $H_{R}$ and $M_{R}$ after recovering from the infection. The susceptible mosquitoes population is denoted $V_{s}$. After transmission occurs from biting an infectious human, susceptible mosquitoes transition to the exposed population, $V_{E}$. The end of extrinsic incubation period marks the exposed mosquitoes shift to the infectious class $V_{I}$, where they remain infectious until death.

For the construction of the model:

- There is immunity in the recovered state, the infected man can infect a woman and a susceptible man (result of the study of other epidemics that are transmitted by sexual contact).

- The death by natural causes is equal in any state, the death of mosquitoes will be due to environmental factors because no control strategy is applied.

- By definition epidemiological $H_{s}, M_{s}, H_{E}, M_{E}, H_{I}, M_{I}, H_{R}, M_{R}, V_{s}, V_{E}$ and $V_{I}$ are continue and positive or null.

The transmission dynamics of the ZIKV is modeled by the following system 
of ordinary differential equations:

$$
\begin{aligned}
\frac{d H_{s}}{d t} & =N_{1}-\beta_{y_{1}} V_{I} H_{s}-\beta_{y_{2}} H_{I} H_{s}-\mu_{1} H_{s}, \\
\frac{d M_{s}}{d t} & =N_{2}-\beta_{y_{1}} V_{I} M_{s}-\beta_{y_{3}} H_{I} M_{s}-\mu_{2} M_{s}, \\
\frac{d H_{E}}{d t} & =\beta_{y_{1}} V_{I} H_{s}+\beta_{y_{2}} H_{I} H_{s}-\left(\omega_{1}+\mu_{1}\right) H_{E}, \\
\frac{d M_{E}}{d t} & =\beta_{y_{1}} V_{I} M_{s}+\beta_{y_{3}} H_{I} M_{s}-\left(\omega_{2}+\mu_{2}\right) M_{E}, \\
\frac{d H_{I}}{d t} & =\omega_{1} H_{E}-\left(\epsilon_{1}+\mu_{1}+r_{1}\right) H_{I}, \\
\frac{d M_{I}}{d t} & =\omega_{2} M_{E}-\left(\epsilon_{2}+\mu_{2}+r_{2}\right) M_{I}, \\
\frac{d H_{R}}{d t} & =r_{1} H_{I}-\mu_{1} H_{R}, \\
\frac{d M_{R}}{d t} & =r_{2} M_{I}-\mu_{2} M_{R}, \\
\frac{d V_{s}}{d t} & =N_{3}-\beta_{x} H_{I} V_{s}-\beta_{x} M_{I} V_{s}-\eta V_{s}, \\
\frac{d V_{E}}{d t} & =\beta_{x} H_{I} V_{s}+\beta_{x} M_{I} V_{s}-\left(\omega_{3}+\eta\right) V_{E}, \\
\frac{d V_{I}}{d t} & =\omega_{3} V_{E}-\eta V_{I} .
\end{aligned}
$$

Initial Conditions

$$
\begin{array}{ccc}
t \in\left[0, t_{f}\right] & \\
H_{s}(0)=h_{s}>0 & M_{s}(0)=m_{s}>0 & H_{I}(0)=h_{i}>0 \\
M_{I}(0)=m_{i}>0 & H_{R}(0)=h_{r} \geq 0 & M_{R}(0)=m_{r} \geq 0 \\
H_{E}(0)=h_{e} \geq 0 & M_{E}(0)=m_{e} \geq 0 & V_{s}(0)=v_{s}>0 \\
V_{I}(0)=v_{i}>0 & V_{E}(0)=v_{e} \geq 0 . & \\
0<N_{1}, N_{2}, N_{3}, \beta_{y_{1}}, \beta_{y_{2}}, \beta_{y_{3}}, \beta_{x}, \mu_{1}, \mu_{2}, \omega_{1}, \omega_{2}, \omega_{3}, \eta, r_{1}, r_{2}, \epsilon_{1}, \epsilon_{2} \leq 1
\end{array}
$$

\section{Model Analysis}

Let:

$$
\begin{gathered}
H_{s}+H_{E}+H_{I}+H_{R}=N, \\
M_{s}+M_{E}+M_{I}+M_{R}=M, \\
V_{s}+V_{E}+V_{I}=V .
\end{gathered}
$$

We begin by showing all feasible solutions are uniformly bounded in a proper subset of $\Omega$. The feasible region $\Omega$ with

$\Omega=\left\{\left(H_{s}, H_{E}, H_{I}, H_{R}, M_{s}, M_{E}, M_{I}, M_{R}, V_{s}, V_{I}, V_{R}\right): N \leq \frac{N_{1}}{\mu_{1}}, M \leq \frac{N_{2}}{\mu_{2}}, V \leq \frac{N_{3}}{\eta}\right\}$

Differentiating both sides of $3.2,3.3$ and 3.4 with appropriate substitutions, we obtained the following differential equations: 


$$
\begin{gathered}
N^{\prime}=N_{1}-\mu_{1} N-\epsilon_{1} H_{I} \leq N_{1}-\mu_{1} N \\
M^{\prime}=N_{2}-\mu_{2} M-\epsilon_{2} M_{I} \leq N_{2}-\mu_{2} M \\
V^{\prime}=N_{3}-\eta V
\end{gathered}
$$

Applying Grönwall Inequality in 3.5, 3.6 and 3.7, we obtained:

$$
\begin{gathered}
N(t) \leq N(0) \exp \left(-\mu_{1} t\right)+\frac{N_{1}}{\mu_{1}}\left(1-\exp \left(-\mu_{1} t\right)\right), \\
M(t) \leq M(0) \exp \left(-\mu_{2} t\right)+\frac{N_{2}}{\mu_{2}}\left(1-\exp \left(-\mu_{2} t\right)\right), \\
V(t) \leq V(0) \exp (-\eta t)+\frac{N_{3}}{\eta}(1-\exp (-\eta t)),
\end{gathered}
$$

where $N(0), M(0)$ and $V(0)$ represents the initial humans and mosquitoes population total.

Therefore, $0 \leq N \leq \frac{N_{1}}{\mu_{1}}, 0 \leq M \leq \frac{N_{2}}{\mu_{2}}$ and $0 \leq V \leq \frac{N_{3}}{\eta}$ as $t \rightarrow \infty$. This implies, $\frac{N_{1}}{\mu_{1}}$ is an upper bound for $N(t), \frac{N_{2}}{\mu_{2}}$ is an upper bound for , $M(t)$ and $\frac{N_{3}}{\eta}$ is an upper bound for $V(t)$ provided $N(0) \leq \frac{N_{1}}{\mu_{1}}, M(0) \leq \frac{N_{2}}{\mu_{2}}$ and $V(0) \leq \frac{N_{3}}{\eta}$

Hence, all feasible solutions of the model system (3.1) enter the region $\Omega$ which is a positively invariant set. Thus, the system is biologically meaningful and mathematically well-posed in the domain of $\Omega$. In this domain, it is sufficient to consider the dynamics of the flow generated by the model system described by (3.1). Therefore, we summarized the results in the following lemma:

Lemma 3.1. The closed set $\Omega$ is positively invariant and attracting with respect to the model described by 3.1 . 


\section{Theorem 3.1. (Existence and Uniqueness Theorem)}

Let:

$$
\begin{aligned}
& f_{1}=\frac{d H_{s}}{d t}=N_{1}-\beta_{y_{1}} V_{I} H_{s}-\beta_{y_{2}} H_{I} H_{s}-\mu_{1} H_{s} ; \quad H_{s}(0)=h_{s}, \\
& f_{2}=\frac{d M_{s}}{d t}=N_{2}-\beta_{y_{1}} V_{I} M_{s}-\beta_{y_{3}} H_{I} M_{s}-\mu_{2} M_{s} ; \quad M_{s}(0)=m_{s}, \\
& f_{3}=\frac{d H_{E}}{d t}=\beta_{y_{1}} V_{I} H_{s}+\beta_{y_{2}} H_{I} H_{s}-\left(\omega_{1}+\mu_{1}\right) H_{E} ; \quad H_{E}(0)=h_{e}, \\
& f_{4}=\frac{d M_{E}}{d t}=\beta_{y_{1}} V_{I} M_{s}+\beta_{y_{3}} H_{I} M_{s}-\left(\omega_{2}+\mu_{2}\right) M_{E} ; \quad M_{E}(0)=m_{e}, \\
& f_{5}=\frac{d H_{I}}{d t}=\omega_{1} H_{E}-\left(\epsilon_{1}+\mu_{1}+r_{1}\right) H_{I} ; \quad H_{I}(0)=h_{i}, \\
& f_{6}=\frac{d M_{I}}{d t}=\omega_{2} M_{E}-\left(\epsilon_{2}+\mu_{2}+r_{1}\right) M_{I} ; \quad M_{I}(0)=m_{i}, \\
& f_{7}=\frac{d H_{R}}{d t}=r_{1} H_{I}-\mu_{1} H_{R} ; \quad H_{R}(0)=h_{r}, \\
& f_{8}=\frac{d M_{R}}{d t}=r_{2} M_{I}-\mu_{2} M_{R} ; \quad M_{R}(0)=m_{r}, \\
& f_{9}=\frac{d V_{s}}{d t}=N_{3}-\beta_{x} H_{I} V_{s}-\beta_{x} M_{I} V_{s}-\eta V_{s} ; \quad V_{s}(0)=v_{s}, \\
& f_{10}=\frac{d V_{E}}{d t}=\beta_{x} H_{I} V_{s}+\beta_{x} M_{I} V_{s}-\left(\omega_{3}+\eta\right) V_{E} ; \quad V_{E}(0)=v_{E}, \\
& f_{11}=\frac{d V_{I}}{d t}=\omega_{3} V_{E}-\eta V_{I} ; \quad V_{I}(0)=v_{I} .
\end{aligned}
$$

$$
\begin{aligned}
& D=\left\{\left(H_{s}, M_{s}, H_{E}, M_{E}, H_{I}, M_{I}, H_{R}, M_{R}, V_{s}, V_{E}, V_{I}\right):\left|t-t_{0}\right| \leq a,\right. \\
& \left|H_{s}-h_{s}\right| \leq b,\left|M_{S}-m_{s}\right| \leq c,\left|H_{I}-h_{i}\right| \leq d,\left|M_{I}-m_{i}\right| \leq e,\left|H_{R}-h_{r}\right| \leq f,
\end{aligned}
$$$$
\left|M_{R}-m_{r}\right| \leq g,\left|H_{E}-h_{e}\right| \leq h,\left|M_{E}-m_{e}\right| \leq i,\left|V_{s}-v_{s}\right| \leq j,\left|V_{E}-v_{e}\right| \leq
$$
$\left.k,\left|V_{I}-v_{i}\right| \leq l\right\}$ then the system (3.8) has a unique solution.

Proof. We calculate the partial derivatives of $f_{i}, i=1,2, \ldots, 11$ respect $x_{j}=H_{s}, M_{s}, H_{E}, M_{E}, H_{I}, M_{I}, H_{R}, M_{R}, V_{s}, V_{E}, V_{I}$, and evaluate at the origin: $\frac{\partial f_{1}}{\partial H_{s}}=-\mu_{1}, \quad \frac{\partial f_{1}}{\partial M_{s}}=\frac{\partial f_{1}}{\partial H_{E}}=\frac{\partial f_{1}}{\partial M_{E}}=\frac{\partial f_{1}}{\partial H_{I}}=\frac{\partial f_{1}}{\partial M_{I}}=\frac{\partial f_{1}}{\partial H_{R}}=\frac{\partial f_{1}}{\partial M_{R}}=$ $\frac{\partial f_{1}}{\partial V_{s}}=\frac{\partial f_{1}}{\partial V_{E}}=\frac{\partial f_{1}}{\partial V_{I}}=0$.

$\frac{\partial f_{2}}{\partial H_{s}}=-\mu_{2}, \quad \frac{\partial f_{2}}{\partial M_{s}}=\frac{\partial f_{2}}{\partial H_{E}}=\frac{\partial f_{2}}{\partial M_{E}}=\frac{\partial f_{2}}{\partial H_{I}}=\frac{\partial f_{2}}{\partial M_{I}}=\frac{\partial f_{2}}{\partial H_{R}}=\frac{\partial f_{2}}{\partial M_{R}}=$ $\frac{\partial f_{2}}{\partial V_{s}}=\frac{\partial f_{2}}{\partial V_{E}}=\frac{\partial f_{2}}{\partial V_{I}}=0$.

$\frac{\partial f_{3}}{\partial H_{E}}=-\left(\omega_{1}+\mu_{1}\right), \quad \frac{\partial f_{3}}{\partial H_{s}}=\frac{\partial f_{3}}{\partial M_{s}}=\frac{\partial f_{3}}{\partial M_{E}}=\frac{\partial f_{3}}{\partial H_{I}}=\frac{\partial f_{3}}{\partial M_{I}}=\frac{\partial f_{3}}{\partial H_{R}}=$ $\frac{\partial f_{3}}{\partial M_{R}}=\frac{\partial f_{3}}{\partial V_{s}}=\frac{\partial f_{3}}{\partial V_{E}}=\frac{\partial f_{3}}{\partial V_{I}}=0$.

$\frac{\partial f_{4}}{\partial M_{E}}=-\left(\omega_{2}+\mu_{2}\right), \quad \frac{\partial f_{4}}{\partial H_{s}}=\frac{\partial f_{4}}{\partial M_{s}}=\frac{\partial f_{4}}{\partial H_{E}}=\frac{\partial f_{4}}{\partial H_{I}}=\frac{\partial f_{4}}{\partial M_{I}}=\frac{\partial f_{4}}{\partial H_{R}}=$ 
$\frac{\partial f_{4}}{\partial M_{R}}=\frac{\partial f_{4}}{\partial V_{s}}=\frac{\partial f_{4}}{\partial V_{E}}=\frac{\partial f_{4}}{\partial V_{I}}=0$.

$\frac{\partial f_{5}}{\partial H_{I}}=-\left(\epsilon_{1}+\mu_{1}+r_{1}\right), \quad \frac{\partial f_{5}}{\partial H_{E}}=\omega_{1}, \quad \frac{\partial f_{5}}{\partial H_{s}}=\frac{\partial f_{5}}{\partial M_{s}}=\frac{\partial f_{5}}{\partial M_{E}}=\frac{\partial f_{5}}{\partial M_{I}}=$ $\frac{\partial f_{5}}{\partial H_{R}}=\frac{\partial f_{5}}{\partial M_{R}}=\frac{\partial f_{5}}{\partial V_{s}}=\frac{\partial f_{5}}{\partial V_{E}}=\frac{\partial f_{5}}{\partial V_{I}}=0$.

$\frac{\partial f_{6}}{\partial M_{E}}=\omega_{2}, \quad \frac{\partial f_{6}}{\partial M_{I}}=-\left(\epsilon_{2}+\mu_{2}+r_{2}\right), \quad \frac{\partial f_{6}}{\partial H_{s}}=\frac{\partial f_{6}}{\partial M_{s}}=\frac{\partial f_{6}}{\partial H_{E}}=\frac{\partial f_{6}}{\partial H_{I}}=$ $\frac{\partial f_{6}}{\partial H_{R}}=\frac{\partial f_{6}}{\partial M_{R}}=\frac{\partial f_{6}}{\partial V_{s}}=\frac{\partial f_{6}}{\partial V_{E}}=\frac{\partial f_{6}}{\partial V_{I}}=0$.

$\frac{\partial f_{7}}{\partial H_{I}}=r_{1}, \quad \frac{\partial f_{7}}{\partial H_{R}}=-\mu_{1}, \quad \frac{\partial f_{7}}{\partial H_{s}}=\frac{\partial f_{7}}{\partial M_{s}}=\frac{\partial f_{7}}{\partial H_{E}}=\frac{\partial f_{7}}{\partial M_{E}}=\frac{\partial f_{7}}{\partial M_{I}}=$ $\frac{\partial f_{7}}{\partial M_{I}}=\frac{\partial f_{7}}{\partial V_{s}}=\frac{\partial f_{7}}{\partial V_{E}}=\frac{\partial f_{7}}{\partial V_{I}}=0$

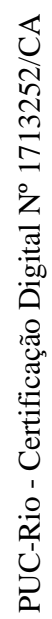

$\frac{\partial f_{8}}{\partial M_{I}}=r_{2}, \quad \frac{\partial f_{8}}{\partial M_{R}}=-\mu_{2}, \quad \frac{\partial f_{8}}{\partial H_{s}}=\frac{\partial f_{8}}{\partial M_{s}}=\frac{\partial f_{8}}{\partial H_{E}}=\frac{\partial f_{8}}{\partial M_{E}}=\frac{\partial f_{8}}{\partial H_{I}}=$ $\frac{\partial f_{8}}{\partial H_{R}}=\frac{\partial f_{8}}{\partial V_{s}}=\frac{\partial f_{8}}{\partial V_{E}}=\frac{\partial f_{8}}{\partial V_{I}}=0$

$\frac{\partial f_{9}}{\partial V_{s}}=-\eta, \quad \frac{\partial f_{9}}{\partial H_{s}}=\frac{\partial f_{9}}{\partial M_{s}}=\frac{\partial f_{9}}{\partial H_{E}}=\frac{\partial f_{9}}{\partial M_{E}}=\frac{\partial f_{9}}{\partial H_{I}}=\frac{\partial f_{9}}{\partial M_{I}}=\frac{\partial f_{9}}{\partial H_{R}}=$ $\frac{\partial f_{9}}{\partial M_{R}}=\frac{\partial f_{9}}{\partial V_{E}}=\frac{\partial f_{9}}{\partial V_{I}}=0$

$\frac{\partial f_{10}}{\partial V_{E}}=-\left(\omega_{3}+\eta\right), \quad \frac{\partial f_{10}}{\partial H_{s}}=\frac{\partial f_{10}}{\partial M_{s}}=\frac{\partial f_{10}}{\partial H_{E}}=\frac{\partial f_{10}}{\partial M_{E}}=\frac{\partial f_{10}}{\partial H_{I}}=\frac{\partial f_{10}}{\partial M_{I}}=$ $\frac{\partial f_{10}}{\partial H_{R}}=\frac{\partial f_{10}}{\partial M_{R}}=\frac{\partial f_{10}}{\partial V_{s}}=\frac{\partial f_{10}}{\partial V_{I}}=0$

$\frac{\partial f_{11}}{\partial V_{E}}=\omega_{3}, \quad \frac{\partial f_{11}}{\partial V_{I}}=-\eta, \quad \frac{\partial f_{11}}{\partial H_{s}}=\frac{\partial f_{11}}{\partial M_{s}}=\frac{\partial f_{11}}{\partial H_{E}}=\frac{\partial f_{11}}{\partial M_{E}}=\frac{\partial f_{11}}{\partial H_{I}}=$ $\frac{\partial f_{11}}{\partial M_{I}}=\frac{\partial f_{11}}{\partial H_{R}}=\frac{\partial f_{11}}{\partial M_{R}}=\frac{\partial f_{11}}{\partial V_{s}}=0$.

Therefore $\left|\frac{\partial f_{i}}{\partial x_{j}}\right|$ are continuous and bounded. Hence, following [11] of Theorem 2.1 above, the problem 3.8 has a unique solution and do the model 3.1 is both epidemiologically feasible and mathematically well posed. 


\section{$\Re_{0}$ and Local Stability}

The disease-free equilibrium of the model is given as:

$$
v_{0}=\left(\frac{N_{1}}{\mu_{1}}, 0,0,0, \frac{N_{2}}{\mu_{2}}, 0,0,0, \frac{N_{3}}{\eta}, 0,0\right) .
$$

To study the $\Re_{0}$ we will divide into two subsystems, in one the study for only sexual contagion and the other for only contagion by mosquito bites, to interpret their influence independently in the spread of the epidemic.

The mosquito transmission route only model is obtained by assuming that virus is not transmitted sexually. The subsystem with only sexual contagion is:

$$
\begin{aligned}
\frac{d H_{s}}{d t} & =N_{1}-\beta_{y_{1}} V_{I} H_{s}-\mu_{1} H_{s}, \\
\frac{d M_{s}}{d t} & =N_{2}-\beta_{y_{1}} V_{I} M_{s}-\mu_{2} M_{s}, \\
\frac{d H_{E}}{d t} & =\beta_{y_{1}} V_{I} H_{s}-\left(\omega_{1}+\mu_{1}\right) H_{E}, \\
\frac{d M_{E}}{d t} & =\beta_{y_{1}} V_{I} M_{s}+-\left(\omega_{2}+\mu_{2}\right) M_{E}, \\
\frac{d H_{I}}{d t} & =\omega_{1} H_{E}-\left(\epsilon_{1}+\mu_{1}+r_{1}\right) H_{I}, \\
\frac{d M_{I}}{d t} & =\omega_{2} M_{E}-\left(\epsilon_{2}+\mu_{2}+r_{2}\right) M_{I}, \\
\frac{d H_{R}}{d t} & =r_{1} H_{I}-\mu_{1} H_{R}, \\
\frac{d M_{R}}{d t} & =r_{2} M_{I}-\mu_{2} M_{R}, \\
\frac{d V_{s}}{d t} & =N_{3}-\beta_{x} H_{I} V_{s}-\beta_{x} M_{I} V_{s}-\eta V_{s}, \\
\frac{d V_{E}}{d t} & =\beta_{x} H_{I} V_{s}+\beta_{x} M_{I} V_{s}-\left(\omega_{3}+\eta\right) V_{E}, \\
\frac{d V_{I}}{d t} & =\omega_{3} V_{E}-\eta V_{I} .
\end{aligned}
$$

where:

$$
T=\left[\begin{array}{cccccc}
0 & 0 & 0 & 0 & 0 & \beta_{y_{1}} H_{s} \\
0 & 0 & 0 & 0 & 0 & 0 \\
0 & 0 & 0 & 0 & 0 & \beta_{y_{1}} M_{s} \\
0 & 0 & 0 & 0 & 0 & 0 \\
0 & \beta_{x} V_{s} & 0 & \beta_{x} V_{s} & 0 & 0 \\
0 & 0 & 0 & 0 & 0 & 0
\end{array}\right]
$$


$\Sigma=\left[\begin{array}{cccccc}-\left(\omega_{1}+\mu_{1}\right) & 0 & 0 & 0 & 0 & 0 \\ 0 & -\left(\epsilon_{1}+r_{1}+\mu_{1}\right) & 0 & 0 & 0 & 0 \\ 0 & 0 & -\left(\omega_{2}+\mu_{2}\right) & 0 & 0 & 0 \\ 0 & 0 & 0 & -\left(\epsilon_{2}+r_{2}+\mu_{2}\right) & 0 & 0 \\ 0 & 0 & 0 & 0 & -\left(\omega_{3}+\eta\right) & 0 \\ 0 & 0 & 0 & 0 & 0 & -\eta\end{array}\right]$,

are the transmission and transition matrices, see Section 2.3.

Using the methodology presented in Section 2.3 (Lemma 2.1) for $v_{0}$, the reproduction number basic is given by:

$$
\Re_{0}^{m}=\rho\left(-T \Sigma^{-1}\right)=\sqrt{k_{1}+k_{2}}
$$

where

$k_{1}=\frac{\beta_{y_{1}} N_{1} \beta_{x} N_{3} \omega_{1} \omega_{3}}{\mu_{1} \eta^{2}\left(\omega_{1}+\mu_{1}\right)\left(\epsilon_{1}+\mu_{1}+r_{1}\right)\left(\omega_{3}+\eta\right)}, k_{2}=\frac{\beta_{y_{1}} N_{2} \beta_{x} N_{3} \omega_{2} \omega_{3}}{\mu_{2} \eta^{2}\left(\omega_{2}+\mu_{2}\right)\left(\epsilon_{2}+\mu_{2}+r_{2}\right)\left(\omega_{3}+\eta\right)}$.

Using Theorem 2.6 you get the following result:

Lemma 3.2. The disease-free equilibrium is locally asymptotically stable if $\Re_{0}^{m}<1$, and unstable if $\Re_{0}^{m}>1$ for the sub-model with only mosquito transmission.

The sexual transmission route only model is obtained by assuming that ZIKA virus is only transmitted sexually and not through the bites of infectious mosquitoes. The model reduces to the following:

$$
\begin{aligned}
\frac{d H_{s}}{d t} & =N_{1}-\beta_{y_{2}} H_{I} H_{s}-\mu_{1} H_{s}, \\
\frac{d M_{s}}{d t} & =N_{2}-\beta_{y_{3}} H_{I} M_{s}-\mu_{2} M_{s}, \\
\frac{d H_{E}}{d t} & =\beta_{y_{2}} H_{I} H_{s}-\left(\omega_{1}+\mu_{1}\right) H_{E}, \\
\frac{d M_{E}}{d t} & =\beta_{y_{3}} H_{I} M_{s}-\left(\omega_{2}+\mu_{2}\right) M_{E} \\
\frac{d H_{I}}{d t} & =\omega_{1} H_{E}-\left(\epsilon_{1}+\mu_{1}+r_{1}\right) H_{I}, \\
\frac{d M_{I}}{d t} & =\omega_{2} M_{E}-\left(\epsilon_{2}+\mu_{2}+r_{2}\right) M_{I}, \\
\frac{d H_{R}}{d t} & =r_{1} H_{I}-\mu_{1} H_{R}, \\
\frac{d M_{R}}{d t} & =r_{2} M_{I}-\mu_{2} M_{R} .
\end{aligned}
$$

The transmission and transition matrices are:

$$
T=\left[\begin{array}{cccc}
0 & \beta_{y_{2}} H_{s} & 0 & 0 \\
0 & 0 & 0 & 0 \\
0 & \beta_{y_{3}} M_{s} & 0 & 0 \\
0 & 0 & 0 & 0
\end{array}\right]
$$




$$
\Sigma=\left[\begin{array}{cccc}
-\left(\omega_{1}+\mu_{1}\right) & 0 & 0 & 0 \\
0 & -\left(\epsilon_{1}+r_{1}+\mu_{1}\right) & 0 & \\
0 & 0 & -\left(\omega_{2}+\mu_{2}\right) & 0 \\
0 & 0 & 0 & -\left(\epsilon_{2}+r_{2}+\mu_{2}\right)
\end{array}\right] .
$$

Using the same methodology for the previous subsystem, the number reproduction basic is:

$$
\Re_{0}^{s}=\rho\left(-T \Sigma^{-1}\right)=\frac{\beta_{y_{2}} N_{1} \omega_{1}}{\mu_{1}\left(\omega_{1}+\mu_{1}\right)\left(\epsilon_{1}+\mu_{1}+r_{1}\right)} .
$$

Using Theorem 2.6 you get the following lemma:

Lemma 3.3. The disease-free equilibrium is locally asymptotically stable if $\Re_{0}^{s}<1$, and unstable if $\Re_{0}^{s}>1$ for the sub-model with only sexual transmission.

\section{2}

\section{Model with Temporal Delay}

The mosquito becomes infected when it consumes the blood of a sick person. Then, if the insect bites a healthy person, it transmits the virus, which enters the bloodstream and is incubated for 3 to 12 days, until the symptom begins appearance. The delay $\tau$ will refer to the time that the mosquito that delays in developing the pathogen, 4 to 7 days $[15,16]$. The delay is taken into account in the infected compartment. The parameters and variables maintain the definitions and restraints of the model 3.1.

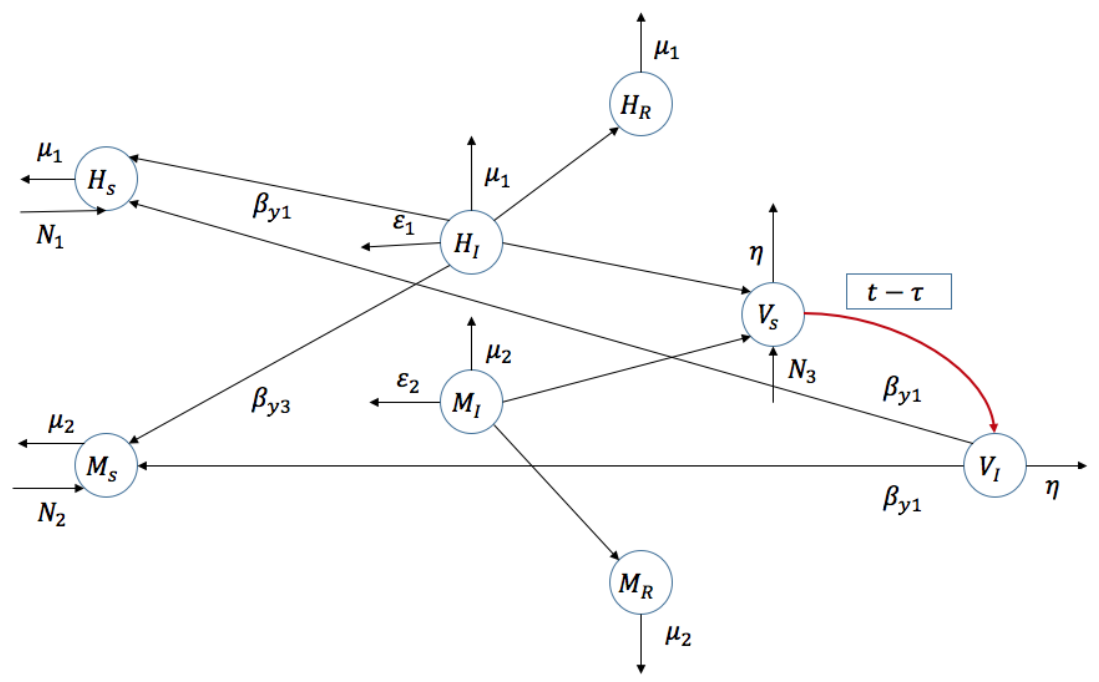

Figure 3.2.1: Schematic Representation of Model with Temporal Delay. 
The transmission dynamics of the ZIKV taking into account the time delay is modeled by the system of differential equations with delay following:

$$
\begin{aligned}
\frac{d H_{s}}{d t} & =N_{1}-\beta_{y_{1}} V_{I} H_{s}-\beta_{y_{2}} H_{I} H_{s}-\mu_{1} H_{s}, \\
\frac{d M_{s}}{d t} & =N_{2}-\beta_{y_{1}} V_{I} M_{s}-\beta_{y_{3}} H_{I} M_{s}-\mu_{2} M_{s}, \\
\frac{d H_{I}}{d t} & =\beta_{y_{1}} V_{I}(t-\tau) H_{s}+\beta_{y_{2}} H_{I} H_{s}-\left(\mu_{1}+r_{1}+\epsilon_{1}\right) H_{I}, \\
\frac{d M_{I}}{d t} & =\beta_{y_{1}} V_{I}(t-\tau) M_{s}+\beta_{y_{3}} H_{I} M_{s}-\left(\mu_{2}+r_{2}+\epsilon_{2}\right) M_{I}, \\
\frac{d H_{R}}{d t} & =r_{1} H_{I}-\mu_{1} H_{R}, \\
\frac{d M_{R}}{d t} & =r_{2} M_{I}-\mu_{2} M_{R}, \\
\frac{d V_{s}}{d t} & =N_{3}-\beta_{x} H_{I} V_{s}-\beta_{x} M_{I} V_{s}-\eta V_{s}, \\
\frac{d V_{I}}{d t} & =\beta_{x} H_{I} V_{s}+\beta_{x} M_{I} V_{s}-\eta V_{I} .
\end{aligned}
$$

Initial Conditions

$$
\begin{array}{ccc}
H_{s}(0)=h_{s}>0 & M_{s}(0)=m_{s}>0 & H_{I}(0)=h_{i}>0 \\
M_{I}(0)=m_{i}>0 & H_{R}(0)=h_{r} \geq 0 & M_{R}(0)=m_{r} \geq 0 \\
V_{s}(0)=v_{s}>0 & V_{I}(0)=v_{i}>0 . \\
{[-\tau, 0] \rightarrow \mathbb{R}^{+}, \quad \tau>0}
\end{array}
$$

\section{Model Analysis}

Let $f(t, x, y)=\left(F_{1}(t, x, y), F_{2}(t, x, y), \ldots, F_{8}(t, x, y)\right)$,

$x=\left(H_{s}, M_{s}, H_{I}, M_{I}, H_{R}, M_{R}, V_{s}, V_{I}\right)$ and $y=V_{I}(t-\tau)$.

$V_{I}(t-\tau)$ is continue and positive for epidemiological definition.

Where:

$$
\begin{aligned}
& F_{1}(t, x, y)=N_{1}-\beta_{y_{1}} V_{I} H_{s}-\beta_{y_{2}} H_{I} H_{s}-\mu_{1} H_{s}, \\
& F_{2}(t, x, y)=N_{2}-\beta_{y_{1}} V_{I} M_{s}-\beta_{y_{3}} H_{I} M_{s}-\mu_{2} M_{s}, \\
& F_{3}(t, x, y)=\beta_{y_{1}} V_{I}(t-\tau) H_{s}+\beta_{y_{2}} H_{I} H_{s}-\left(\mu_{1}+r_{1}+\epsilon_{1}\right) H_{I}, \\
& F_{4}(t, x, y)=\beta_{y_{1}} V_{I}(t-\tau) M_{s}+\beta_{y_{3}} H_{I} M_{s}-\left(\mu_{2}+r_{2}+\epsilon_{2}\right) M_{I}, \\
& F_{5}(t, x, y)=r_{1} H_{I}-\mu_{1} H_{R}, \\
& F_{6}(t, x, y)=r_{2} M_{I}-\mu_{2} M_{R}, \\
& F_{7}(t, x, y)=N_{3}-\beta_{x} H_{I} V_{s}-\beta_{x} M_{I} V_{s}-\eta V_{s}, \\
& F_{8}(t, x, y)=\beta_{x} H_{I} V_{s}+\beta_{x} M_{I} V_{s}-\eta V_{I} .
\end{aligned}
$$

$F_{i}(t, x, y), i=1,2, . ., 8$ are continues, then $f(t, x, y)$ is continue.

$$
\begin{aligned}
& \frac{\partial F_{1}}{\partial H_{s}}=-\beta_{y_{1}} V_{I}-\beta_{y_{2}} H_{I}-\mu_{1}, \quad \frac{\partial F_{1}}{\partial H_{I}}=-\beta_{y_{2}} H_{s}, \quad \frac{\partial F_{1}}{\partial V_{I}}=-\beta_{y_{1}} H_{s}, \\
& \frac{\partial F_{1}}{\partial M_{s}}=\frac{\partial F_{1}}{\partial M_{I}}=\frac{\partial F_{1}}{\partial H_{R}}=\frac{\partial F_{1}}{\partial M_{R}}=\frac{\partial F_{1}}{\partial V_{s}}=0 .
\end{aligned}
$$




$$
\begin{aligned}
& \frac{\partial F_{2}}{\partial M_{s}}=-\beta_{y_{1}} V_{I}-\beta_{y_{3}} H_{I}-\mu_{2}, \quad \frac{\partial F_{2}}{\partial H_{I}}=-\beta_{y_{3}} M_{s}, \quad \frac{\partial F_{2}}{\partial V_{I}}=-\beta_{y_{1}} M_{s} \\
& \frac{\partial F_{2}}{\partial H_{s}}=\frac{\partial F_{2}}{\partial M_{I}}=\frac{\partial F_{2}}{\partial H_{R}}=\frac{\partial F_{2}}{\partial M_{R}}=\frac{\partial F_{2}}{\partial V_{s}}=0 .
\end{aligned}
$$

$\frac{\partial F_{3}}{\partial H_{s}}=\beta_{y_{1}} V_{I}(t-\tau)+\beta_{y_{2}} H_{I}, \quad \frac{\partial F_{3}}{\partial H_{I}}=\beta_{y_{2}} H_{s}-\left(\mu_{1}+r_{1}+\epsilon_{1}\right)$,

$\frac{\partial F_{3}}{\partial M_{s}}=\frac{\partial F_{3}}{\partial M_{I}}=\frac{\partial F_{3}}{\partial H_{R}}=\frac{\partial F_{3}}{\partial M_{R}}=\frac{\partial F_{3}}{\partial V_{s}}=0, \frac{\partial F_{3}}{\partial V_{I}}=0$

$\frac{\partial F_{4}}{\partial M_{s}}=\beta_{y_{1}} V_{I}(t-\tau)+\beta_{y_{3}} H_{I}, \quad \frac{\partial F_{4}}{\partial H_{I}}=\beta_{y_{3}} M_{s}, \quad \frac{\partial F_{4}}{\partial M_{I}}=-\left(\mu_{2}+r_{2}+\epsilon_{2}\right)$, $\frac{\partial F_{4}}{\partial H_{s}}=\frac{\partial F_{4}}{\partial H_{R}}=\frac{\partial F_{4}}{\partial M_{R}}=\frac{\partial F_{4}}{\partial V_{s}}=\frac{\partial F_{4}}{\partial V_{I}}=0$

$\frac{\partial F_{5}}{\partial H_{I}}=r_{1}, \quad \frac{\partial F_{5}}{\partial H_{R}}=-\mu_{1}$,

$\frac{\partial F_{5}}{\partial H_{s}}=\frac{\partial F_{5}}{\partial M_{I}}=\frac{\partial F_{5}}{\partial M_{s}}=\frac{\partial F_{5}}{\partial M_{R}}=\frac{\partial F_{5}}{\partial V_{I}}=0$

$\frac{\partial F_{6}}{\partial M_{I}}=r_{2}, \quad \frac{\partial F_{6}}{\partial M_{R}}=-\mu_{2}$

$\frac{\partial F_{6}}{\partial H_{s}}=\frac{\partial F_{6}}{\partial M_{s}}=\frac{\partial F_{6}}{\partial H_{I}}=\frac{\partial F_{6}}{\partial H_{R}}=\frac{\partial F_{6}}{\partial V_{s}}=\frac{\partial F_{6}}{\partial V_{I}}=0$.

$\frac{\partial F_{7}}{\partial H_{I}}=-\beta_{x} V_{s}, \quad \frac{\partial F_{7}}{\partial M_{I}}=-\beta_{x} V_{s}, \quad \frac{\partial F_{7}}{\partial V_{s}}=-\beta_{x} H_{I}-\beta_{x} M_{I}-\eta$,

$\frac{\partial F_{7}}{\partial H_{s}}=\frac{\partial F_{7}}{\partial M_{s}}=\frac{\partial F_{7}}{\partial H_{R}}=\frac{\partial F_{7}}{\partial M_{R}}=\frac{\partial F_{7}}{\partial V_{I}}=0$.

$\frac{\partial F_{8}}{\partial H_{I}}=\beta_{x} V_{s}, \quad \frac{\partial F_{8}}{\partial M_{I}}=\beta_{x} V_{s}, \quad \frac{\partial F_{8}}{\partial V_{s}}=\beta_{x}\left(H_{I}+M_{I}\right), \quad \frac{\partial F_{8}}{\partial V_{I}}=-\eta$,

$\frac{\partial F_{8}}{\partial H_{s}}=\frac{\partial F_{8}}{\partial M_{s}}=\frac{\partial F_{8}}{\partial H_{R}}=\frac{\partial F_{8}}{\partial M_{R}}=0$

$\left(F_{i}\right)_{x} i=1,2, \ldots, 8$ are continues then $f_{x}$ is continue.

The initial conditions are continuous and positive according to the epidemiological definition (number of people), so by Theorem 2.2 the solution of the model is unique.

$F_{1}\left(0, M_{s}, H_{I}, M_{I}, H_{R}, M_{R}, V_{s}, V_{I}\right)=N_{1}>0$,

$F_{2}\left(H_{s}, 0, H_{I}, M_{I}, H_{R}, M_{R}, V_{s}, V_{I}\right)=N_{2}>0$,

$F_{3}\left(H_{s}, M_{s}, 0, M_{I}, H_{R}, M_{R}, V_{s}, V_{I}\right)=\beta_{y_{1}} V_{I}(t-\tau) H_{s} \geq 0$, 
$F_{4}\left(H_{s}, M_{s}, H_{I}, 0, H_{R}, M_{R}, V_{s}, V_{I}\right)=\beta_{y_{1}} V_{I}(t-\tau) M_{s}+\beta_{y_{3}} H_{I} M_{s} \geq 0$,

$F_{5}\left(H_{s}, M_{s}, H_{I}, M_{I}, 0, M_{R}, V_{s}, V_{I}\right)=r_{1} H_{I} \geq 0$,

$F_{6}\left(H_{s}, M_{s}, H_{I}, M_{I}, H_{R}, 0, V_{s}, V_{I}\right)=r_{2} M_{I} \geq 0$,

$F_{7}\left(H_{s}, M_{s}, H_{I}, M_{I}, H_{R}, M_{R}, 0, V_{I}\right)=N_{3}>0$,

$F_{8}\left(H_{s}, M_{s}, H_{I}, M_{I}, H_{R}, M_{R}, V_{s}, 0\right)=\beta_{x} V_{s}\left(H_{I}+M_{I}\right) \geq 0$.

By Theorem 2.4 the solution of the model is positive.

\section{3}

\section{Numerical Simulations}

The objective of this subsection is to compare the two modeling techniques, with respect to the behavior of infected humans. Computational experimentation was carried out for Suriname and El Salvador because they are countries with characteristics that the ZIKV can become an endemic problem. The values of the parameters and initial conditions for each country and their origin are presented in the Tables 3.2, 3.3 and 3.4 and the delay $(\tau)$ is equal to 7 days. It was used for the codes Matlab2017a and to solve the system of ordinary differential equations, an adaptation of the ODE45, which is based on an explicit Runge-Kutta formula, the DormandPrince pair. It is a one-step solver -for the solution in moment $n$, it needs only the solution at the immediately preceding time point, $n-1$. In general, ode45 is the best function to apply as a first try for most problems (https://www.mathworks.com/help/matlab/ref/ode45.html) and for the model with temporary delay, an adaptation of the routine DDE23, it tracks the discontinuities and integrates with the explicit pair of Runge-Kutta $(2,3)$ and the interpolator of ode23. It uses iteration to take steps longer than the delays (https://www .mathworks.com/help/matlab/ref/dde23.html?s_ tid=doc_ta). 


\begin{tabular}{||l|ll||}
\hline Parameters & Value & Reference \\
\hline$\beta_{y_{1}}$ & 0.1705 & {$[33]$} \\
\hline$\beta_{x}$ & 0.4352 & {$[33]$} \\
\hline$\beta_{y_{2}}$ & 0.003 & Assumed \\
\hline$\beta_{y_{3}}$ & 0.003 & Assumed \\
\hline$\omega_{3}$ & $\frac{1}{10.2}$ & {$[36]$} \\
\hline$\omega_{1}=\omega_{2}$ & $\frac{1}{6}$ & {$[6,19]$} \\
\hline$\mu_{1}=\mu_{2}$ & 0.0061 & https://www.indexmundi.com/g/g.aspx?c=ns\&v=26\&l=es \\
\hline$r_{1}=r_{2}$ & 0.55 & Assumed \\
\hline$\epsilon_{1}=\epsilon_{2}$ & 0.0004 & Assumed \\
\hline$\eta$ & $\frac{1}{18}$ & {$[28]$} \\
\hline$N_{1}$ & 0.65 & Assumed \\
\hline$N_{2}$ & 0.75 & Assumed \\
\hline$N_{3}$ & 0.60 & Assumed \\
\hline
\end{tabular}

Table 3.2: Suriname, 2016.

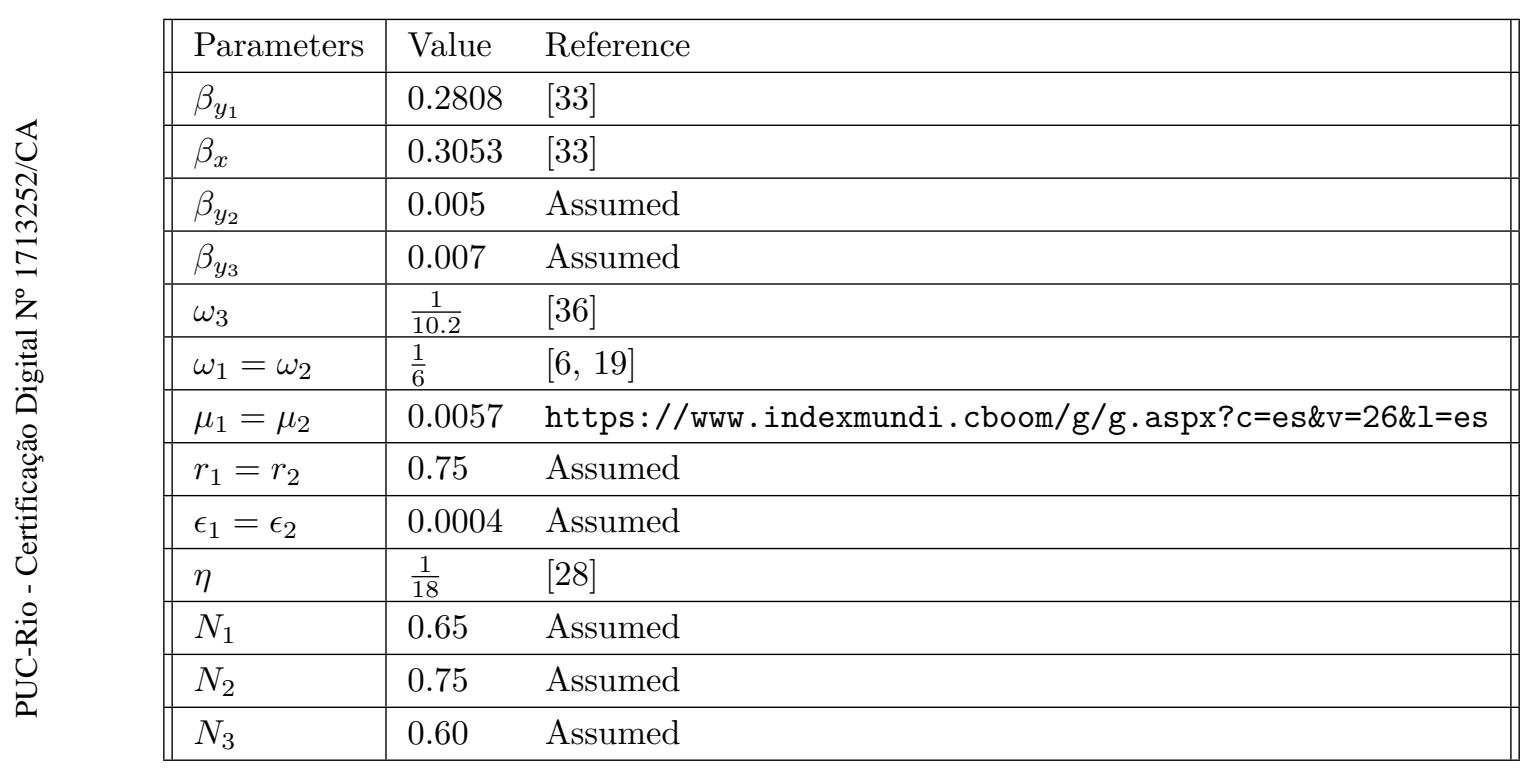

Table 3.3: El Salvador, 2016.

\begin{tabular}{lcll}
\hline Variables & Suriname & El Salvador & Reference \\
\hline$H_{s}$ & 280.298 & 2.982 .221 & $\begin{array}{l}\text { https://www.datosmacro.com/demografia/poblacion/surinam } \\
\text { (el-salvador) }\end{array}$ \\
\hline$M_{S}$ & 278.070 & 3.362 .501 & $\begin{array}{l}\text { https://www.datosmacro.com/demografia/poblacion/el-salvador } \\
\text { (surinam) }\end{array}$ \\
\hline$H_{E}$ & 0 & 0 & Assumed \\
\hline$M_{E}$ & 0 & 0 & Assumed \\
\hline$H_{I}$ & 1000 & 1000 & Assumed \\
\hline$M_{I}$ & 1000 & 1000 & Assumed \\
\hline$H_{R}$ & 0 & 0 & Assumed \\
\hline$M_{R}$ & 0 & 0 & Assumed \\
\hline$V_{s}$ & 44222 & 152362 & {$[33]$} \\
\hline$V_{E}$ & 0 & 0 & Assumed \\
\hline$V_{I}$ & 1000 & 1000 & Assumed \\
\hline
\end{tabular}

Table 3.4: Initial Conditions, 2016. 


\section{$\Re_{0}$ Study}

We found the $\Re_{0}^{m}$ for $\beta_{y_{1 *}} \in[0.04287,1.1241]$ (95\% CI) in Suriname and $\beta_{y_{1 *}} \in[0.0119,0.9244]$ (95\% CI) in El Salvador, the data is extracted from [33]. The minimum value is 3.3932 and 6.3882 for El Salvador and Suriname respectively and shows that the infection will be able to spread in a population. The Figure 3.3.1 show the growth of $\Re_{0}^{m}$ with respect to $\beta_{y_{1}}$ for Suriname and El Salvador.

The value of $\Re_{0}^{s}$ (only with sexual contact) for Suriname and El Salvador (for the values in the Tables 3.1, 3.2 and 3.4 ) shows that the infection will disappear in the long term $\Re_{0}^{s}<1$. This form of contagion does not have a great influence on the spread of the epidemic.
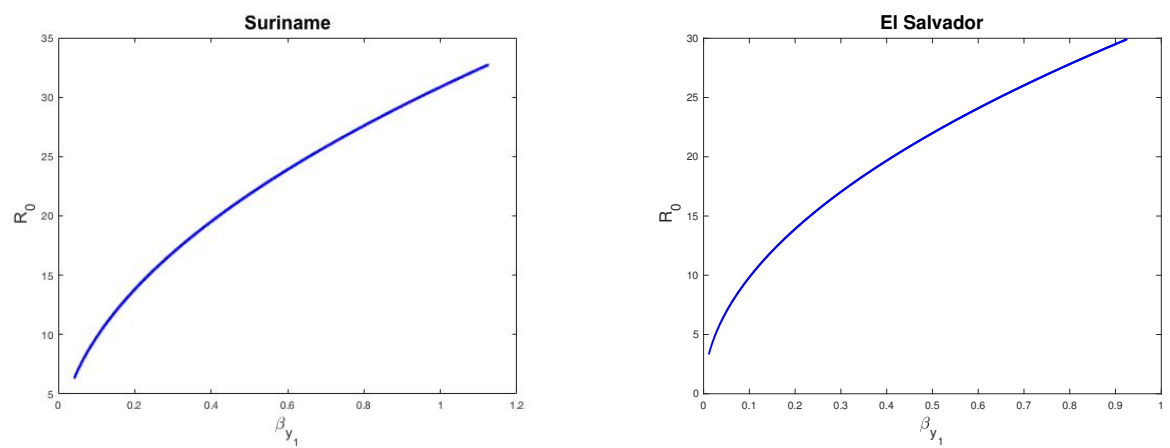

Figure 3.3.1: $\Re_{0}^{m}$

\section{Suriname:}

The model with temporal delay reports a greater number of infected people in the period in which the epidemic has greater force than the model with the exposed variable, see Figure 3.3.2.

During a time close to 45 days both models report the same number of infected (for first time), see figure 3.3.3, but at the end of a year model 3.11 reports a greater number of infected. Throughout the year, the epidemic continues, demonstrating the endemic nature of the epidemic in this country, see Figure 3.3.4. 

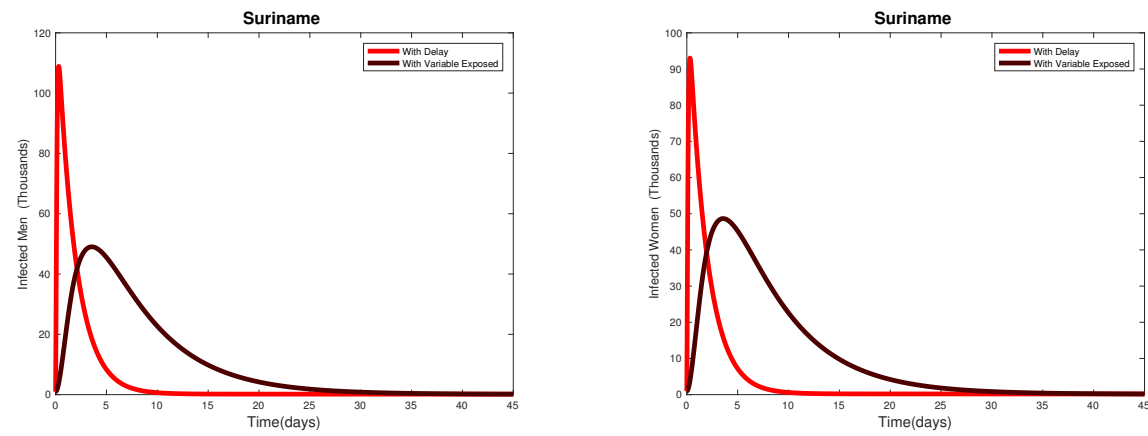

Figure 3.3.2: Comparison between models. Suriname Infected.
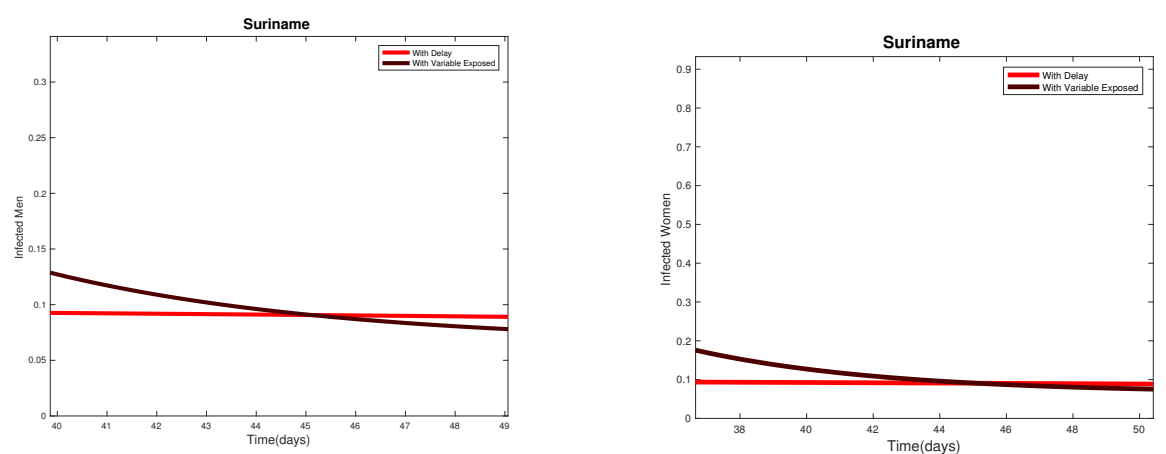

Figure 3.3.3: First Moment of Coincidence. Suriname infected.
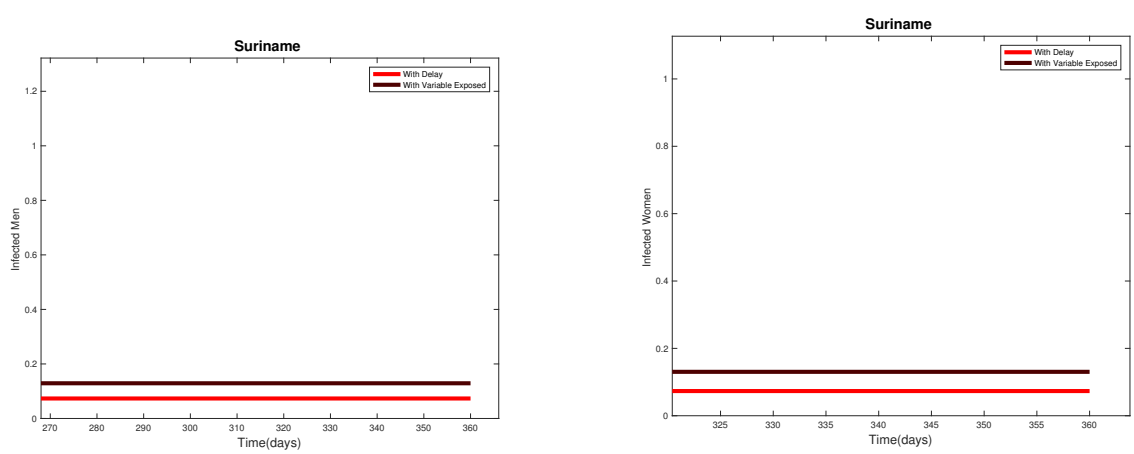

Figure 3.3.4: Infected humans at 360 days. Suriname.

\section{El Salvador:}

The asymptotic behavior of infected humans is analogous to that of Suriname, see Figure 3.3.5. Both models report the same number of infected (for first time) after 50 days, see figure 3.3.6 and the result obtained in the study of $\Re_{0}$ is verified because the ZIKV behaves as endemic, but the opposite occurs Suriname because at the end of the period the model with delay reports 
a greater number of infected people compared to the model with an exposed variable, see Figure 3.3.7.
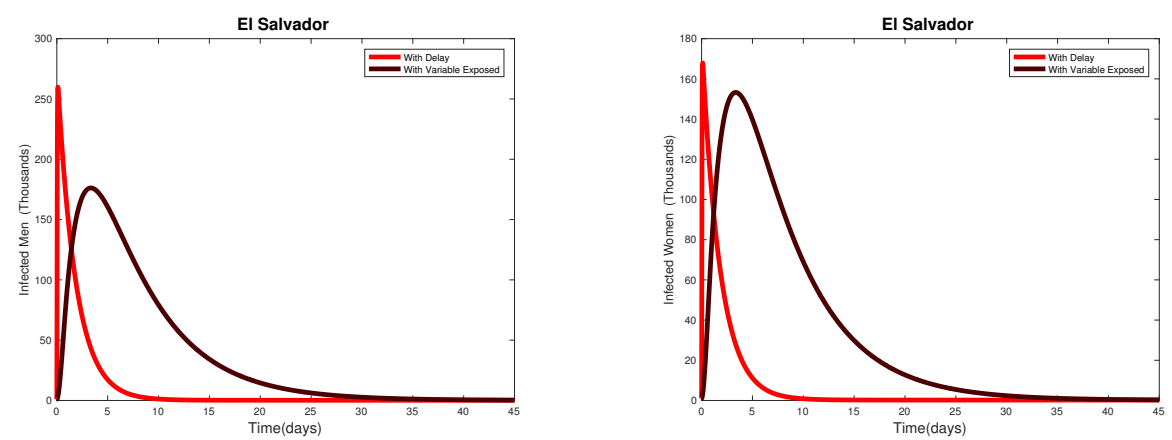

Figure 3.3.5: Comparison between models. El Salvador Infected.
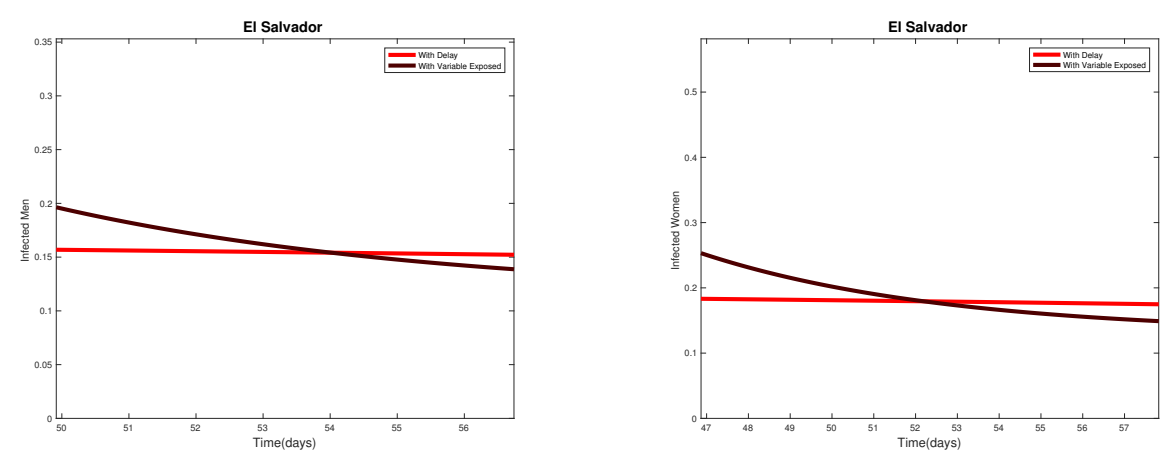

Figure 3.3.6: First Moment of Coincidence. El Salvador infected.
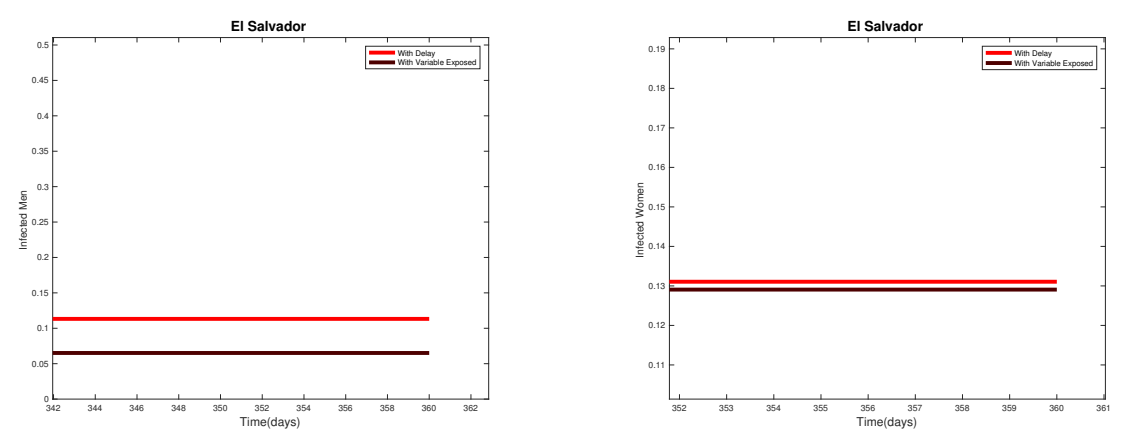

Figure 3.3.7: Infected humans at 360 days. El Salvador. 


\section{4 \\ Preliminary work on diffusive-advective model for spatial evolution of ZIKV epidemic}

The objective of this chapter is to present a model for the Zika epidemic based on the diffusion-advection equations. To solve this model we use a numerical scheme based on the finite elements method (FEM) and finite differences. The computational experimentation and the deep study of the numerical scheme will be the objective of future works.

\section{1 \\ Background}

\section{Advection-Diffusion Equation}

The advection-diffusion equation combines diffusion and advection operators which describe physical phenomena where particles, energy, or other physical quantities are transferred inside a physical system due to two processes: diffusion and advection.

The general equation is [37]:

$$
\frac{\partial c}{\partial t}=\nabla \cdot(D \nabla c)-\nabla \cdot(\vec{v} c)+R
$$

where

$-c$ is the variable of interest.

- $D$ is the diffusivity (called diffusion coefficient).

$-\vec{v}$ is the velocity field that the quantity is moving with. It is function of time and space.

- $R$ describe sources or sinks of the quantity $c$, describes the creation or destruction of the quantity. For example, if $c$ is the concentration of a molecule, then $R$ describes how the molecule can be created or destroyed by chemical reaction, $R$ may be a function and of $c$ of other parameters.

- The $\nabla \cdot(D \nabla c)$ describe diffusion. The net diffusion is proportional to de Laplacian (or second derivative) of concentration if the diffusivity $D$ is a constant. 
- The $-\nabla \cdot(\vec{v} c)$, describes advection.

Typically, the model can be simplified so that it has constant diffusion coefficient, there are no sources of sinks, and the velocity field describes an incompressible flow. Then the formula simplifies to:

$$
\frac{\partial c}{\partial t}=D \nabla^{2} c-\vec{v} \cdot \nabla c
$$

The advection-diffusion equation combines both parabolic and hyperbolic effects which make the model difficult to simulate.

The stationary advection-diffusion equation describes the steady-state behavior of an advective-diffusive system. In steady-state, $\frac{\partial c}{\partial t}=0$, so the formula is:

$$
0=\nabla(D \nabla c)-\nabla \cdot(\vec{v} c)+R
$$

\section{Galerkin Method}

Galerkin methods are a class of methods for converting a continuous operator problem (such as differential equation) to a discrete problem [9, 22, 34].

Let us introduce Galerkin's method with an abstract problem posed as weak formulation on a Hilbert space $V$, namely, find $u \in V$ such that for all $v \in V$, $a(u, v)=f(v)$.

Here, $a(\cdot, \cdot)$ is a bilinear form (the exact requirements on $a(\cdot, \cdot)$ will be specified later) and $f$ is a bounded linear functional on $V$.

Choose a subspace $V_{n} \subset V$ of dimension $n$ and solve the projected problem:

find $u_{n} \in V_{n}$ such that for all $v_{n} \in V_{n}, a\left(u_{n}, v_{n}\right)=f\left(v_{n}\right)$. We call this the Galerkin equation.

Notice that the equation has remained unchanged and only the spaces have changed. Reducing the problem to a finite-dimensional vector subspace allows us to numerically compute $u_{n}$ as a finite linear combination of the basis vectors in $V_{n}$.

The key property of the Galerkin approach is that the error is orthogonal to the chosen subspaces. Since $V_{n} \subset V$, we can use $v_{n}$ as a test vector in the original equation. Subtracting the two, we get the Galerkin orthogonality relation for the error, $\epsilon_{n}=u-u_{n}$ which is the error between the solution of the original problem $u$, and the solution of the Galerkin equation $u_{n}$,

$$
a\left(\epsilon_{n}, v_{n}\right)=a\left(u, v_{n}\right)-a\left(u_{n}, v_{n}\right)=f\left(v_{n}\right)-f\left(v_{n}\right)=0 .
$$

Since the aim of Galerkin's method is the production of a linear system of equations, we build its matrix form, which can be used to compute the solution 
by a computer program.

Let $e_{1}, e_{2}, \ldots, e_{n}$ be a basis for $V_{n}$. Then, it is sufficient to use these in turn for testing the Galerkin equation, find $u_{n} \in V_{n}$ such that $a\left(u_{n}, e_{i}\right)=f\left(e_{i}\right) \quad i=$ $1,2, \ldots, n$.

We expand $u_{n}$ with respect to this basis, $u_{n}=\sum_{j=1}^{n} u_{j} e_{j}$ and insert it into the equation above, to obtain

$$
a\left(\sum_{j=1}^{n} u_{j} e_{j}, e_{i}\right)=\sum_{j=1}^{n} u_{j} a\left(e_{j}, e_{i}\right)=f\left(e_{i}\right) \quad i=1,2, \ldots, n .
$$

This previous equation is actually a linear system of equations $A u=f$, where

$$
A_{i j}=a\left(e_{j}, e_{i}\right), \quad f_{i}=f\left(e_{i}\right)
$$

Due to the definition of the matrix entries, the matrix of the Galerkin equation is symmetric if and only if the bilinear form $a(\cdot, \cdot)$ is symmetric.

\section{Finite Difference and Crank-Nicolson method}

The finite difference method is one of several techniques for obtaining numerical solution to partial difference equation [32]. In all numerical solutions, the continuous partial differential equation (PDE) is replaced with a discrete approximation. In this context, the word discrete means that the numerical solution is known only at a finite number of points in the physical domain. The number of those points can be selected by the user of the numerical method. In general, increasing the number of points not only increases the resolution, but also the accuracy of the numerical solution [38].

The discrete approximation results in a set of algebraic equations that are evaluated (or solve) for the values of the discrete unknowns.

The mesh is the set of locations where the discrete solution is computed. These points are called nodes, and if one were to draw lines between adjacent nodes in the domain the resulting image would resemble a net or mesh. Two key parameters of the mesh are $\Delta x$, the local distance between adjacent points in space, and $\Delta t$, the local distance between adjacent time steps [38].

The core idea of the finite-difference method is to replace continuous derivatives with so-called difference formulas that involve only the discrete values associated with positions on the mesh.

Applying the finite-difference methods to a differential equation involves replacing all derivatives with difference formulas. In the heat equation, there are derivatives with respect to time and derivatives with respect to space. Using different combinations of mesh points in the difference formulas results in the 
different schemes. In the limit as the mesh spacing $(\Delta x$ and $\Delta t)$ go to zero, the numerical solution obtained with any useful scheme will approach the true solution to the original differential equation. However, the rate at which the numerical solution approaches the true solution varies with the scheme. In addition, there are some practically useful schemes that can fail to yield a solution for bad combinations of $\Delta x$ and $\Delta t[38]$.

\section{The Discrete Mesh}

The finite difference method obtains an approximate solution for $\xi(x, t)$ at a finite set $x$ and $t$.

For uniformly spaced in the interval $0 \leq x \leq L$ such that

$$
x_{i}=(i-1) \Delta x, \quad i=1,2, \ldots, N
$$

where $N$ is the total number of spatial nodes, including, those on the boundary.

Given $L$ and $N$, the spacing between the $x_{i}$ is computed with

$$
\Delta x=\frac{L}{N-1} .
$$

Similarly, the discrete $t$ are uniformly spaced in $0 \leq t \leq t_{\max }$ :

$$
t_{m}=(m-1) \Delta t \quad m=1,2, \ldots, M
$$

where $M$ is the number of time steps and $\Delta t$ is the size of a time step

$$
\Delta t=\frac{t_{\max }}{M-1}
$$

\section{First Order Difference [38]}

Consider a Taylor series expansion $\xi(x)$ about the point $x_{i}$

$$
\xi\left(x_{i}+\Delta x\right)=\xi\left(x_{i}\right)+\left.\Delta x \frac{\partial \xi}{\partial x}\right|_{x_{i}}+\left.\frac{(\Delta x)^{2}}{2} \frac{\partial^{2} \xi}{\partial x^{2}}\right|_{x_{i}}+\left.\frac{(\Delta x)^{3}}{3 !} \frac{\partial^{3} \xi}{\partial x^{3}}\right|_{x_{i}}+\ldots
$$

where $\delta x$ is a change in $x$ relative to $x_{i}$. Let $k=\Delta x$ and $h=\Delta t$ and consider the value of $\xi$ at the location of the $x_{i+1}$ mesh line

$$
\xi\left(x_{i}+k\right)=\xi\left(x_{i}\right)+\left.k \frac{\partial \xi}{\partial x}\right|_{x_{i}}+\left.\frac{k^{2}}{2} \frac{\partial^{2} \xi}{\partial x^{2}}\right|_{x_{i}}+\left.\frac{k^{3}}{3 !} \frac{\partial^{3} \xi}{\partial x^{3}}\right|_{x_{i}}+\ldots
$$


Solve for $\left(\frac{\partial \xi}{\partial x}\right)_{x_{i}}$

$$
\left.\frac{\partial \xi}{\partial x}\right|_{x_{i}}=\frac{\xi\left(x_{i}+k\right)-\xi\left(x_{i}\right)}{k}-\left.\frac{k}{2} \frac{\partial^{2} \xi}{\partial x^{2}}\right|_{x_{i}}-\left.\frac{k^{2}}{3 !} \frac{\partial^{3} \xi}{\partial x^{3}}\right|_{x_{i}}+\ldots
$$

Notice that the powers of $\Delta x$ multiplying the partial derivatives on the right hand side have been reduced by one.

Substitute the approximate solution for the exact solution, use $\xi_{i} \approx \xi\left(x_{i}\right)$ and $\xi_{i+1} \approx \xi\left(x_{i}+\Delta x\right)$.

$$
\left.\frac{\partial \xi}{\partial x}\right|_{x_{i}} \approx \frac{\xi_{i+1}-\xi_{i}}{k}-\left.\frac{k}{2} \frac{\partial^{2} \xi}{\partial x^{2}}\right|_{x_{i}}-\left.\frac{k^{2}}{3 !} \frac{\partial^{3} \xi}{\partial x^{3}}\right|_{x_{i}}+\ldots
$$

The mean value theorem can be used to replace the higher order derivatives (exactly)

$$
\left.\frac{k^{2}}{2} \frac{\partial^{2} \xi}{\partial x^{2}}\right|_{x_{i}}+\left.\frac{k^{3}}{3 !} \frac{\partial^{3} \xi}{\partial x^{3}}\right|_{x_{i}}+\ldots=\left.\frac{k^{2}}{2} \frac{\partial^{2} \xi}{\partial x^{2}}\right|_{\sigma}
$$

where $x_{i} \leq \sigma \leq x_{i+1}$. Thus

$$
\left.\frac{\partial \xi}{\partial x}\right|_{x_{i}}-\left.\frac{\xi_{i+1}-\xi_{i}}{k} \approx \frac{k^{2}}{2} \frac{\partial^{2} \xi}{\partial x^{2}}\right|_{\sigma}
$$

The term on the right-hand side of Equation (4.8) is called the truncation error of the finite difference approximation. It is the error that results from truncating the series in Equation (4.7).

In general, $\sigma$ is not known. Note that the right-hand side of Equation (4.8) contain the mesh parameter $\Delta x$, which is chosen by the person using the finite difference simulation. Since this is the only parameter under the user's control that determines the error, the truncation error simply written

$$
\frac{k^{2}}{2} \frac{\partial^{2} \xi}{\partial x^{2}}=O\left(k^{2}\right)
$$

The equals sign in this expression is true in the order of magnitude sense. In other words the $=O\left(k^{2}\right)$ on the right-hand side of the expression is not a strict equality. Rather, the expression means that the left hand side is a product of an unknown constant and $k^{2}$. Although the expression does not give us the exact magnitude of $\left.\frac{k^{2}}{2}\left(\left.\frac{\partial^{2} \xi}{\partial x^{2}}\right|_{x_{i}}\right)\right|_{\sigma}$, it tells us how quickly that term approaches zero as $\Delta x$ is reduced.

Using big $O$ notation, Equation (4.7) can be written

$$
\left.\frac{\partial \xi}{\partial x}\right|_{x_{i}}=\frac{\xi_{i+1}-\xi_{i}}{k}+O(k)
$$


The Equation (4.9) is called the forward difference formula for $\left.\frac{\partial \xi}{\partial x}\right|_{x_{i}}$ because it involves nodes $x_{i}$ and $x_{i+1}$. The forward difference approximation has a truncation error that is $O(k)$.

An alternative first-order finite difference formula is obtained if the Taylor series like that in Equation (4.6) for $-k$. Using the discrete mesh variables in place of all the unknowns, one obtains

$$
\xi_{i-1}=\xi_{i}-\left.k \frac{\partial \xi}{\partial x}\right|_{x_{i}}+\left.\frac{k^{2}}{2} \frac{\partial^{2} \xi}{\partial x^{2}}\right|_{x_{i}}-\left.\frac{k^{3}}{3 !} \frac{\partial^{3} \xi}{\partial x^{3}}\right|_{x_{1}}+\ldots
$$

Using big $O$ notation

$$
\left.\frac{\partial \xi}{\partial x}\right|_{x_{i}}=\frac{\xi_{i}-\xi_{i-1}}{k}+O(k)
$$

This is called the backward difference formula because it involves the values of $\xi$ at $x_{i}$ and $x_{i-1}$.

The order of magnitude of the truncation error for the backward difference approximation is the same as that of the forward difference approximation. Write the Taylor series expansions for $\xi_{i+1}$ and $\xi_{i-1}$ and subtracting, then

$$
\left.\frac{\partial \xi}{\partial x}\right|_{x_{i}}=\frac{\xi_{i+1}-\xi_{i-1}}{2 k}+O\left(k^{2}\right) .
$$

This is the central difference approximation to $\left.\frac{\partial \xi}{\partial x}\right|_{x_{i}}$. To get good approximations to the continuous problem small $k$ is chosen. When $k \leq 1$, the truncation error for the central difference approximation goes to zero much faster than the truncation error backward or forward difference.

Finite difference approximations to higher order derivatives can be obtained with the additional manipulations of Taylor series expansion about $\xi\left(x_{i}\right)$.

$$
\left.\frac{\partial^{2} \xi}{\partial x^{2}}\right|_{x_{i}}=\frac{\xi_{i+1}-2 \xi_{i}+\xi_{i-1}}{k^{2}}+O\left(k^{2}\right)
$$

This is also called the central difference approximation, but (obviously) it is the approximation to the second derivative.

Approximate the time derivative in Equation (4.6) with a forward difference

$$
\left.\frac{\partial \xi}{\partial t}\right|_{\left(t_{m+1}, x_{i}\right)}=\frac{\xi_{i}^{m+1}-\xi_{i}^{m}}{\Delta t}+O(h) .
$$

Note that terms on the right-hand side only involve $\xi$ at $x=x_{i}$.

Use the central difference approximation to $\left.\frac{\partial^{2} \xi}{\partial x^{2}}\right|_{x_{i}}$ and evaluate all terms at the time $m$. 


$$
\left.\frac{\partial^{2} \xi}{\partial x^{2}}\right|_{x_{i}}=\frac{\xi_{i-1}^{m}-2 \xi_{i}^{m}+\xi_{i+1}^{m}}{k^{2}}+O\left(k^{2}\right)
$$

Substitute Equation (4.13) into the left-hand side of Equation (4.6); substitute Equation (4.14) into the right-hand side of Equation (4.6); and collect the truncation error terms to get

$$
\frac{\xi_{i}^{m+1}-\xi_{i}^{m}}{h}=\alpha \frac{\xi_{i-1}^{m}-2 \xi_{i}^{m}+\xi_{i+1}^{m}}{k^{2}}+O(h)+O\left(k^{2}\right)
$$

The temporal errors and the spatial errors have different orders. Also notice that we can explicitly solve for $\xi_{i}^{m+1}$ in terms of the other values of $\xi$. Drop the truncation error terms from Equation (4.15) and solve for $\xi_{i}^{m+1}$ to get

$$
\xi_{i}^{m+1}=\xi_{i}^{m}+\alpha \frac{h}{k^{2}}\left(\xi_{i+1}^{m}-2 \xi_{i}^{m}+\xi_{i-1}^{m}\right)
$$

The Equation (4.16) is called the Forward Time, Centered Space or FTCS approximation to the heat equation.

In the derivation of Equation (4.16), the forward difference was used to approximate the time derivative on the left-hand side of Equation (4.6). Now, choose the backward difference,

$$
\left.\frac{\partial \xi}{\partial t}\right|_{\left(t_{m+1}, x_{i}\right)}=\frac{\xi_{i}^{m}-\xi_{i}^{m-1}}{h}+O(h)
$$

Substitute Equation (4.17) into the left hand side of Equation (4.6); substitute Equation (4.14) into the right-hand side of Equation (4.6); and collect the truncation error terms to get

$$
\frac{\xi_{i}^{m}-\xi_{i}^{m-1}}{h}=\alpha \frac{\xi_{i-1}^{m}-2 \xi_{i}^{m}+\xi_{i+1}^{m}}{k^{2}}+O(h)+O\left(k^{2}\right) .
$$

The Equation (4.18) is called the Backward Time, Centered Space or BTCS. The truncation errors in this approximation have the same order of magnitude as the truncation errors in Equation (4.15).

\section{Crank-Nicolson Method}

The FTCS and BTCS schemes have a temporal truncation error of $O(\Delta t)$. When time-accurate solutions are important, the Crank-Nicolson scheme has significant advantages. The Crank-Nicolson scheme is not significantly more difficult to implement than the BTCS scheme, and it has a temporal truncation error that is $O\left(h^{2}\right)$. The Crank- Nicolson scheme is implicit, like BTCS, it is also unconditional stable.

The left-hand side of the heat equation is approximated with the backward 
time difference used in the BTCS scheme, Equation (4.17). The right-hand side of the heat equation is approximated with the average of the central difference schema evaluated at the current and the previous time step. Thus Equation (4.6) is approximated with

$$
\frac{\xi_{i}^{m}-\xi_{i}^{m-1}}{h}=\frac{\alpha}{2}\left(\frac{\xi_{i-1}^{m}-2 \xi_{i}^{m}+\xi_{i+1}^{m}}{k^{2}}+\frac{\xi_{i-1}^{m-1}-2 \xi_{i}^{m-1}+\xi_{i+1}^{m-1}}{k^{2}}\right) .
$$

Notice that values of $\xi$ from time step $m$ and time step $m-1$ appear on the right-hand side. Equation (4.19) is used to predict the values of $\xi$ at time $m$, so all values of $\xi$ at time $m-1$ are assumed to be known.

The Crank-Nicolson scheme is implicit, and as a result a system of equations for the $\xi$ must be solved at each time step, has a truncation error of $O\left(h^{2}\right)+O\left(k^{2}\right)$ [10].

\section{2}

\section{Advection-Diffusion Model for spatial evolution of ZIKV epidemic}

Consider $S(t, x), I(t, x)$, and $R(t, x)$ functions representing populations of susceptible, infected and recovered humans at time $t>0$ and location $x \in \Omega \subset R^{2}$. Also, consider $M(t, x)$ and $P(t, x)$ the populations of susceptible and infected mosquitoes. In this model for the ZIKV epidemic, the infection is only due to the bites of mosquitoes infected with the virus, and it is not necessary to stratify the population by sex. Assumptions:

- We accept immunity: from the recovered state does not possible to return to the susceptible state.

- There is not consideration of vertical transmission in humans or mosquitoes.

- The death by natural causes is equal in any state.

- The death of mosquitoes will be due to environmental factors because no control strategy is applied.

- The Laplacian $(\Delta)$ and the gradient $(\nabla)$ operators are relative to the spatial variable $x \in \Omega$.

- Assume that the initial data are continuous and bounded functions on $\bar{\Omega}$. 
The formulation of the model is:

$$
\begin{gathered}
\frac{\partial S}{\partial t}-\nabla \cdot\left(\alpha_{s} \nabla S\right)+\nabla \cdot\left(\beta_{s} S\right)=N_{1}-\beta_{y_{1}} S P-\mu S, \\
\frac{\partial I}{\partial t}-\nabla \cdot\left(\alpha_{I} \nabla I\right)+\nabla \cdot\left(\beta_{I} I\right)=\beta_{y_{1}} S P-(r+\epsilon+\mu) I, \\
\frac{\partial R}{\partial t}-\nabla \cdot\left(\alpha_{r} \nabla R\right)+\nabla \cdot\left(\beta_{r} R\right)=r I-\mu R, \\
\frac{\partial M}{\partial t}-\nabla \cdot\left(\alpha_{m} \nabla M\right)+\nabla \cdot\left(\beta_{m} M\right)=N_{4}-\beta_{x} M I-\xi M, \\
\frac{\partial P}{\partial t}-\nabla \cdot\left(\alpha_{p} \nabla P\right)+\nabla \cdot\left(\beta_{p} P\right)=\beta_{x} M I-\xi P . \\
t \in\left[0, t_{f}\right],
\end{gathered}
$$

Initial conditions:

$$
\begin{gathered}
S(0, x)=s_{0}(x)>0, \quad I(0, x)=i_{0}(x)>0, \quad R(0, x)=r_{0}(x) \geq 0, \\
M(0, x)=l_{0}(x) \geq 0, P(0, x)=p_{0}(x) \geq 0, \quad x \in \Omega .
\end{gathered}
$$

Boundary condition (Zero influx conditions):

$$
\begin{gathered}
\frac{\partial S\left(t, x^{*}\right)}{\partial \eta}=\frac{\partial I\left(t, x^{*}\right)}{\partial \eta}=\frac{\partial R\left(t, x^{*}\right)}{\partial \eta}=\frac{\partial M\left(t, x^{*}\right)}{\partial \eta}=\frac{\partial P\left(t, x^{*}\right)}{\partial \eta}=0, \quad x^{*} \in \partial \Omega . \\
0<\alpha_{s}, \alpha_{I}, \alpha_{r}, \alpha_{m}, \alpha_{p}, \beta_{s}, \beta_{I}, \beta_{r}, \beta_{m}, \beta_{p}, N_{1}, N_{4}, \beta_{y_{1}}, r, \mu, \epsilon, \beta_{x}, \xi \leq 1 .
\end{gathered}
$$

The homogeneous Neumann boundary conditions mean that there is no population flux across the boundary $\partial \Omega$ and both the human and mosquito individuals live in a self-contained environment. The $\eta$ is the outward normal vector to $\partial \Omega$.

The elements of the model are described in the below Table (4.1).

\begin{tabular}{|l|l|}
\hline Parameters & Description \\
\hline$\alpha_{s}$ & Dispersion rate of susceptible humans \\
\hline$\alpha_{I}$ & Dispersion rate of infected humans \\
\hline$\alpha_{r}$ & Dispersion rate of recovered humans \\
\hline$\alpha_{m}$ & Dispersion rate of susceptible mosquitoes \\
\hline$\alpha_{p}$ & Dispersion rate of infected mosquitoes \\
\hline$\beta_{s}$ & Advective transport rate of susceptible humans \\
\hline$\beta_{I}$ & Advective transport rate of infected humans \\
\hline$\beta_{r}$ & Advective transport rate of recovered humans \\
\hline$\beta_{m}$ & Advective transport rate of susceptible mosquitoes \\
\hline$\beta_{p}$ & Advective transport rate of infected mosquitoes \\
\hline$\beta_{x}$ & The force of infection from infected human to susceptible mosquito \\
\hline$\beta_{y_{1}}$ & The force of infection from infected mosquito to susceptible human \\
\hline$\mu, \xi$ & Human and mosquito natural death rates \\
\hline$\epsilon$ & Disease-induced death rate for humans \\
\hline$r$ & Human recovery rate \\
\hline$N_{1}$ & Entry rate of susceptible humans \\
\hline$N_{4}$ & Entry rate of susceptible mosquitoes \\
\hline
\end{tabular}

Table 4.1: Description of parameters used in the model 4.20. 
The right member of the system of equations is represented by the following scheme:

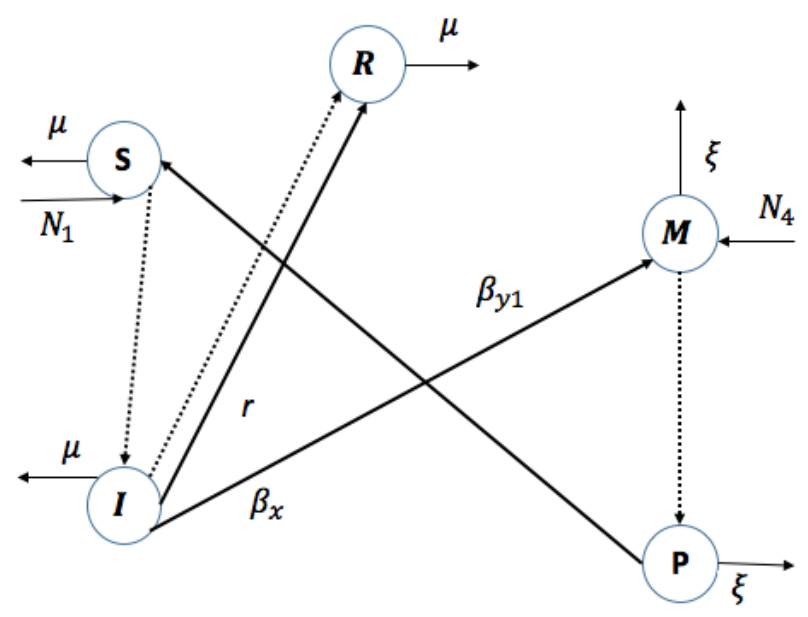

Figure 4.2.1: Schematic representation of the diffusion model.

In Figure 4.2.1 we present a schematic interpretation of the right side of equations 4.20 (the transmission of ZIKV in human and mosquito populations). Susceptible humans start in S and move to I, the infected population, once infected by a mosquito carrying the virus and sexual contact. Infectious humans will then move to and remain in $\mathrm{R}$ after recovering from the infection. The susceptible mosquitoes population is denoted M. After transmission occurs from biting an infectious human, susceptible mosquitoes transition to the infected population, $\mathrm{P}$, where they remain infectious until death. Dashed lines represent the transition of states.)

The $\beta_{s}, \beta_{I}$ and $\beta_{r}$ are the velocities field relative to the migratory movement of susceptible, infected and recovered humans, respectively.

We will consider the mosquito dispersal as the result of a random (and local) flying movement, macroscopically represented by a diffusion process with coefficients $\alpha_{m}$ and $\alpha_{p}$, coupled to a wind advection caused by a constants velocity flux $\beta_{m}$ and $\beta_{p}$. Constant advection can be justified as a 'bias' in the transport process caused by a long-term geographical direction of the wind, while its random and short-term fluctuations are to be included in the diffusion term.

This model can be adapted to any epidemic transmitted by mosquitoes. 
Chapter 4. Preliminary work on diffusive-advective model for spatial evolution of ZIKV epidemic

\section{3}

\section{Method of solution using FEM / Crank-Nicolson}

First we find the variational formulation of the model and apply the Galerkin method, $[9,10]$.

Let $W=\left\{L^{2}\left(\left[0, t_{f}\right]\right), V\right\}$ space of solutions with $V=H^{1}(\Omega)=\{v \in$ $\left.L^{2}(\Omega) \mid \nabla v \in L^{2}(\Omega)\right\}$ space of test functions and in $V$ we define the scalar product:

$$
\langle u, v\rangle=\int_{\Omega} u v d x d y, \quad\langle\nabla u|| \nabla v\rangle=\int_{\Omega} \nabla u \nabla v d \mu
$$

$u \in W, v \in V$.

Multiply by $v$

$$
\begin{aligned}
& \left\langle\frac{\partial S}{\partial t}, v\right\rangle-\alpha_{s}\langle\Delta S, v\rangle+\beta_{s}\langle\nabla S, v\rangle=N_{1}-\frac{\beta_{y_{1}} c_{1}}{V}\langle S P, v\rangle-\mu\langle S, v\rangle, \\
& \left\langle\frac{\partial I}{\partial t}, v\right\rangle-\alpha_{I}\langle\Delta I, v\rangle+\beta_{I}\langle\nabla I, v\rangle=\frac{\beta_{y_{1}} c_{1}}{V}\langle S P, v\rangle-(\epsilon+\mu)\langle I, v\rangle, \\
& \left\langle\frac{\partial R}{\partial t}, v\right\rangle-\alpha_{r}\langle\Delta R, v\rangle+\beta_{r}\langle\nabla R, v\rangle=r\langle I, v\rangle-\mu\langle R, v\rangle, \\
& \left\langle\frac{\partial M}{\partial t}, v\right\rangle-\alpha_{m}\langle\Delta M, v\rangle+\beta_{m}\langle\nabla M, v\rangle=N_{4}-\frac{c_{1} \beta_{x}}{P}\langle M I, v\rangle-\xi\langle M, v\rangle, \\
& \left\langle\frac{\partial P}{\partial t}, v\right\rangle-\alpha_{p}\langle\Delta P, v\rangle+\beta_{p}\langle\nabla P, v\rangle=\frac{c_{1} \beta_{x}}{P}\langle M I, v\rangle-\xi\langle P, v\rangle .
\end{aligned}
$$

Let $U=S, I, R, M, P, U(t, x, y)=U, v=v(x, y),(x, y) \in \Omega$, by the formula of Green

$$
-\alpha_{U}\langle\Delta U, v\rangle=\alpha_{U}\langle\nabla U \| \nabla v\rangle-\alpha_{U}\left\langle\frac{\partial U}{\partial \eta}, v\right\rangle
$$

and for border conditions, $\frac{\partial U}{\partial \eta}=0$ in $\partial \Omega$, we have

$$
-\alpha_{U}\langle\Delta U, v\rangle=\alpha_{U}\langle\nabla U \| \nabla v\rangle
$$

We write the parameters (constants) as follows:

$$
\beta_{s}=\left\langle\beta_{s_{1}}, \beta_{s_{2}}\right\rangle, \quad \beta_{I}=\left\langle\beta_{I_{1}}, \beta_{I_{2}}\right\rangle, \quad \beta_{r}=\left\langle\beta_{r_{1}}, \beta_{r_{2}}\right\rangle, \quad \beta_{m}=\left\langle\beta_{m_{1}}, \beta_{m_{2}}\right\rangle, \quad \beta_{p}=\left\langle\beta_{p_{1}}, \beta_{p_{2}}\right\rangle .
$$


Then,

$$
\begin{aligned}
& \left\langle\frac{\partial S}{\partial t}, v\right\rangle+\alpha_{s}\langle\nabla S|| \nabla v\rangle+\beta_{s_{1}}\left\langle\frac{\partial S}{\partial x}, v\right\rangle+\beta_{s_{2}}\left\langle\frac{\partial S}{\partial y}, v\right\rangle=N_{1}-\frac{\beta_{y_{1}} c_{1}}{V}\langle S P, v\rangle-\mu\langle S, v\rangle, \\
& \left\langle\frac{\partial I}{\partial t}, v\right\rangle+\alpha_{I}\langle\nabla I|| \nabla v\rangle+\beta_{I_{1}}\left\langle\frac{\partial I}{\partial x}, v\right\rangle+\beta_{I_{2}}\left\langle\frac{\partial I}{\partial y}, v\right\rangle=\frac{\beta_{y_{1}} c_{1}}{V}\langle S P, v\rangle-(\epsilon+\mu)\langle I, v\rangle, \\
& \left\langle\frac{\partial R}{\partial t}, v\right\rangle+\alpha_{r}\langle\nabla R \| \nabla v\rangle+\beta_{r_{1}}\left\langle\frac{\partial R}{\partial x}, v\right\rangle+\beta_{r_{2}}\left\langle\frac{\partial R}{\partial y}, v\right\rangle=r\langle I, v\rangle-\mu\langle R, v\rangle, \\
& \left\langle\frac{\partial M}{\partial t}, v\right\rangle+\alpha_{m}\langle\nabla M \| \nabla v\rangle+\beta_{m_{1}}\left\langle\frac{\partial M}{\partial x}, v\right\rangle+\beta_{m_{2}}\left\langle\frac{\partial M}{\partial y}, v\right\rangle=N_{4}-\frac{c_{1} \beta_{x}}{P}\langle M I, v\rangle-\xi\langle M, v\rangle, \\
& \left\langle\frac{\partial P}{\partial t}, v\right\rangle+\alpha_{p}\langle\nabla P \| \nabla v\rangle+\beta_{p_{1}}\left\langle\frac{\partial P}{\partial x}, v\right\rangle+\beta_{p_{2}}\left\langle\frac{\partial P}{\partial y}, v\right\rangle=\frac{c_{1} \beta_{x}}{P}\langle M I, v\rangle-\xi\langle P, v\rangle .
\end{aligned}
$$

Let $\left\{\phi_{i}\right\}_{i=1}^{n}$ base of $V_{h} \subset V$ be a finite approximation of the problem. We have to find $S_{j}, I_{j}, R_{j}, M_{j}$ and $P_{j}$ and we use the notation

$$
U_{h}=\sum_{j=1}^{n} U_{j}(t) \phi_{j}(x, y) .
$$

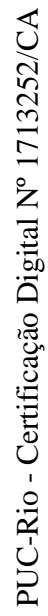

The respective derivatives are:

$$
\begin{gathered}
\frac{\partial U_{h}}{\partial t}=\sum_{j=1}^{n} \frac{d U_{j}}{d t} \phi_{j}(x, y), \\
\frac{\partial U_{h}}{\partial x}=\sum_{j=1}^{n} U_{j} \frac{\partial \phi_{j}}{\partial x}, \\
\frac{\partial U_{h}}{\partial y}=\sum_{j=1}^{n} U_{j} \frac{\partial \phi_{j}}{\partial y} .
\end{gathered}
$$

We have the system

$$
\begin{aligned}
& \sum_{j} \frac{d S_{j}}{d t}\left\langle\phi_{j}, v\right\rangle+\alpha_{s} \sum_{j} S_{j}\left\langle\nabla \phi_{j}|| \nabla v\right\rangle+\beta_{s_{1}} \sum_{j} S_{j}\left\langle\frac{\partial \phi_{j}}{\partial x}, v\right\rangle+\beta_{s_{2}} \sum_{j} S_{j}\left\langle\frac{\partial \phi_{j}}{\partial y}, v\right\rangle= \\
& N_{1}-\frac{\beta_{y_{1}} c_{1}}{V} \sum_{j} P_{j} \sum_{k} S_{k}\left\langle\phi_{j} \phi_{k}, v\right\rangle-\mu \sum_{j} S_{j}\left\langle\phi_{j}, v\right\rangle, \\
& \sum_{j} \frac{d I_{j}}{d t}\left\langle\phi_{j}, v\right\rangle+\alpha_{I} \sum_{j} I_{j}\left\langle\nabla \phi_{j}|| \nabla v\right\rangle+\beta_{I_{1}} \sum_{j} I_{j}\left\langle\frac{\partial \phi_{j}}{\partial x}, v\right\rangle+\beta_{I_{2}} \sum_{j} I_{j}\left\langle\frac{\partial \phi_{j}}{\partial y}, v\right\rangle= \\
& \frac{\beta_{y_{1}} c_{1}}{V} \sum_{j} P_{j} \sum_{k} S_{k}\left\langle\phi_{j} \phi_{k}, v\right\rangle-(\mu+\epsilon) \sum_{j} I_{j}\left\langle\phi_{j}, v\right\rangle,
\end{aligned}
$$




$$
\begin{aligned}
& \sum_{j} \frac{d R_{j}}{d t}\left\langle\phi_{j}, v\right\rangle+\alpha_{r} \sum_{j} R_{j}\left\langle\nabla \phi_{j}|| \nabla v\right\rangle+\beta_{r_{1}} \sum_{j} R_{j}\left\langle\frac{\partial \phi_{j}}{\partial x}, v\right\rangle+\beta_{r_{2}} \sum_{j}\left\langle\frac{\partial \phi_{j}}{\partial y}, v\right\rangle= \\
& r \sum_{j} I_{j}\left\langle\phi_{j}, v\right\rangle-\mu \sum_{j} R_{j}\left\langle\phi_{j}, v\right\rangle, \\
& \sum_{j} M_{j}\left\langle\phi_{j}, v\right\rangle+\alpha_{m} \sum_{j} M_{j}\left\langle\nabla \phi_{j}|| \nabla v\right\rangle+\beta_{m_{1}} \sum_{j} M_{j}\left\langle\frac{\partial \phi_{j}}{\partial x}, v\right\rangle+\beta_{m_{2}} \sum_{j} M_{j}\left\langle\frac{\partial \phi_{j}}{\partial y}, v\right\rangle= \\
& N_{4}-\frac{c_{1} \beta_{x}}{P} \sum_{j} I_{j} \sum_{k} M_{k}\left\langle\phi_{j} \phi_{k}, v\right\rangle-\xi \sum_{j} M_{j}\left\langle\phi_{j}, v\right\rangle, \\
& \sum_{j} P_{j}\left\langle\phi_{j}, v\right\rangle+\alpha_{p} \sum_{j} P_{j}\left\langle\nabla \phi_{j}|| \nabla v\right\rangle+\beta_{p_{1}} \sum_{j} P_{j}\left\langle\frac{\partial \phi_{j}}{\partial x}, v\right\rangle+\beta_{p_{2}} \sum_{j} p_{j}\left\langle\frac{\partial \phi_{j}}{\partial y}, v\right\rangle= \\
& \frac{c_{1} \beta_{x}}{P} \sum_{j} I_{j} \sum_{k} M_{k}\left\langle\phi_{j} \phi_{k}, v\right\rangle-\xi \sum_{j} p_{j}\left\langle\phi_{j}, v\right\rangle .
\end{aligned}
$$

Applying $v \in V_{h}$ we obtain the system:

$$
\begin{aligned}
& \sum_{j} \frac{d S_{j}}{d t}\left\langle\phi_{j}, \phi_{i}\right\rangle+\alpha_{s} \sum_{j} S_{j}\left\langle\nabla \phi_{j}|| \nabla \phi_{i}\right\rangle+\beta_{s_{1}} \sum_{j} S_{j}\left\langle\frac{\partial \phi_{j}}{\partial x}, \phi_{i}\right\rangle+\beta_{s_{2}} \sum_{j} S_{j}\left\langle\frac{\partial \phi_{j}}{\partial y}, \phi_{i}\right\rangle \\
& =N_{1}-\beta_{y_{1}} \sum_{j} P_{j} \sum_{k} S_{k}\left\langle\phi_{j} \phi_{k}, \phi_{i}\right\rangle-\mu \sum_{j} S_{j}\left\langle\phi_{j}, \phi_{i}\right\rangle, \\
& \sum_{j} \frac{d I_{j}}{d t}\left\langle\phi_{j}, \phi_{i}\right\rangle+\alpha_{I} \sum_{j} I_{j}\left\langle\nabla \phi_{j}|| \nabla \phi_{i}\right\rangle+\beta_{I_{1}} \sum_{j} I_{j}\left\langle\frac{\partial \phi_{j}}{\partial x}, \phi_{i}\right\rangle+\beta_{I_{2}} \sum_{j} I_{j}\left\langle\frac{\partial \phi_{j}}{\partial y}, \phi_{i}\right\rangle \\
& =\beta_{y_{1}} \sum_{j} P_{j} \sum_{k} S_{k}\left\langle\phi_{j} \phi_{k}, \phi_{i}\right\rangle-(r+\mu+\epsilon) \sum_{j} I_{j}\left\langle\phi_{j}, v\right\rangle, \\
& \sum_{j} \frac{d R_{j}}{d t}\left\langle\phi_{j}, \phi_{i}\right\rangle+\alpha_{r} \sum_{j} R_{j}\left\langle\nabla \phi_{j} \| \nabla \phi_{i}\right\rangle+\beta_{r_{1}} \sum_{j} R_{j}\left\langle\frac{\partial \phi_{j}}{\partial x}, \phi_{i}\right\rangle+\beta_{r_{2}} \sum_{j} R_{j}\left\langle\frac{\partial \phi_{j}}{\partial y}, \phi_{i}\right\rangle \\
& =r \sum_{j} I_{j}\left\langle\phi_{j}, \phi_{i}\right\rangle-\mu \sum_{j} R_{j}\left\langle\phi_{j}, v\right\rangle, \\
& \sum_{j} M_{j}\left\langle\phi_{j}, v\right\rangle+\alpha_{m} \sum_{j} M_{j}\left\langle\nabla \phi_{j}|| \nabla \phi_{i}\right\rangle+\beta_{m_{1}} \sum_{j} M_{j}\left\langle\frac{\partial \phi_{j}}{\partial x}, v\right\rangle+\beta_{m_{2}} \sum_{j} M_{j}\left\langle\frac{\partial \phi_{j}}{\partial y}, \phi_{i}\right\rangle \\
& =N_{4}-\beta_{x} \sum_{j} I_{j} \sum_{k} M_{k}\left\langle\phi_{j} \phi_{k}, \phi_{i}\right\rangle-\xi \sum_{j} M_{j}\left\langle\phi_{j}, \phi_{i}\right\rangle, \\
& \sum_{j} P_{j}\left\langle\phi_{j}, \phi_{i}\right\rangle+\alpha_{p} \sum_{j} P_{j}\left\langle\nabla \phi_{j} \| \nabla \phi_{i}\right\rangle+\beta_{p_{1}} \sum_{j} P_{j}\left\langle\frac{\partial \phi_{j}}{\partial x}, \phi_{i}\right\rangle+\beta_{p_{2}} \sum_{j} p_{j}\left\langle\frac{\partial \phi_{j}}{\partial y}, \phi_{i}\right\rangle \\
& =\beta_{x} \sum_{j} I_{j} \sum_{k} M_{k}\left\langle\phi_{j} \phi_{k}, \phi_{i}\right\rangle-\xi \sum_{j} p_{j}\left\langle\phi_{j}, \phi_{i}\right\rangle .
\end{aligned}
$$

For the temporary variables the Crank-Nicolson method (central differences in the time $t_{\frac{n+1}{2}}$ ) was used

$$
\begin{aligned}
& \frac{U_{j}}{d t}\left(x, y, t_{n+\frac{1}{2}}\right)=\frac{U_{j}^{n+1}-U_{j}^{n}}{2} . \\
& \frac{d U_{j}}{d t}\left(x, y, t_{n+\frac{1}{2}}\right)=\frac{U_{j}^{n+1}-U_{j}^{n}}{\Delta t},
\end{aligned}
$$


and the scheme is, [7],

$$
\begin{aligned}
& \sum_{j}\left(\frac{S_{j}^{n+1}-S_{j}^{n}}{\Delta t}\right)\left\langle\phi_{j}, \phi_{i}\right\rangle+\alpha_{s} \sum_{j}\left(\frac{S_{j}^{n+1}+S_{j}^{n}}{2}\right)\left\langle\nabla \phi_{j} \| \nabla \phi_{i}\right\rangle+\beta_{s_{1}} \sum_{j}\left(\frac{S_{j}^{n+1}+S_{j}^{n}}{2}\right)\left\langle\frac{\partial \phi_{j}}{\partial x}, \phi_{i}\right\rangle \\
& +\beta_{s_{2}} \sum_{j}\left(\frac{S_{j}^{n+1}+S_{j}^{n}}{2}\right)\left\langle\frac{\partial \phi_{j}}{\partial y}, \phi_{i}\right\rangle \beta_{s_{2}} \sum_{j}\left(\frac{S_{j}^{n+1}+S_{j}^{n}}{2}\right)\left\langle\frac{\partial \phi_{j}}{\partial y}, \phi_{i}\right\rangle-\mu \sum_{j}\left(\frac{S_{j}^{n+1}+S_{j}^{n}}{2}\right)\left\langle\phi_{j}, \phi_{i}\right\rangle . \\
& \sum_{j}\left(\frac{I_{j}^{n+1}-I_{j}^{n}}{\Delta t}\right)\left\langle\phi_{j}, \phi_{i}\right\rangle+\alpha_{I} \sum_{j}\left(\frac{I_{j}^{n+1}+I_{j}^{n}}{2}\right)\left\langle\nabla \phi_{j} \| \nabla \phi_{i}\right\rangle+\beta_{I_{1}} \sum_{j}\left(\frac{I_{j}^{n+1}+I_{j}^{n}}{2}\right)\left\langle\frac{\partial \phi_{j}}{\partial x}, \phi_{i}\right\rangle \\
& +\beta_{I_{2}} \sum_{j}\left(\frac{I_{j}^{n+1}+I_{j}^{n}}{2}\right)\left\langle\frac{\partial \phi_{j}}{\partial y}, \phi_{i}\right\rangle=\beta_{y_{1}} \sum_{j}\left(\frac{P_{j}^{n+1}+P_{j}^{n}}{2}\right) \sum_{k}\left(\frac{S_{k}^{n+1}+S_{k}^{n}}{2}\right)\left\langle\phi_{j} \phi_{k}, \phi_{i}\right\rangle \\
& -(r+\mu+\epsilon) \sum_{j}\left(\frac{I_{j}^{n+1}+I_{j}^{n}}{2}\right)\left\langle\phi_{j}, \phi_{i}\right\rangle . \\
& \sum_{j}\left(\frac{R_{j}^{n+1}-R_{j}^{n}}{\Delta t}\right)\left\langle\phi_{j}, \phi_{i}\right\rangle+\alpha_{r} \sum_{j}\left(\frac{R_{j}^{n+1}+R_{j}^{n}}{2}\right)\left\langle\nabla \phi_{j}|| \nabla \phi_{i}\right\rangle+\beta_{r_{1}} \sum_{j}\left(\frac{R_{j}^{n+1}+R_{j}^{n}}{2}\right)\left\langle\frac{\partial \phi_{j}}{\partial x}, \phi_{i}\right\rangle \\
& +\beta_{r_{2}} \sum_{j}\left(\frac{R_{j}^{n+1}+R_{j}^{n}}{2}\right)\left\langle\frac{\partial \phi_{j}}{\partial y}, \phi_{i}\right\rangle=r \sum_{j}\left(\frac{I_{j}^{n+1}+I_{j}^{n}}{2}\right)\left\langle\phi_{j}, \phi_{i}\right\rangle-\mu \sum_{j}\left(\frac{R_{j}^{n+1}+R_{j}^{n}}{2}\right)\left\langle\phi_{j}, \phi_{i}\right\rangle . \\
& \sum_{j}\left(\frac{M_{j}^{n+1}-M_{j}^{n}}{\Delta t}\right)\left\langle\phi_{j}, \phi_{i}\right\rangle+\alpha_{m} \sum_{j}\left(\frac{M_{j}^{n+1}+M_{j}^{n}}{2}\right)\left\langle\nabla \phi_{j} \| \nabla \phi_{i}\right\rangle+\beta_{m_{1}} \sum_{j}\left(\frac{M_{j}^{n+1}+M_{j}^{n}}{2}\right)\left\langle\frac{\partial \phi_{j}}{\partial x}, \phi_{i}\right\rangle \\
& +\beta_{m_{2}} \sum_{j}\left(\frac{M_{j}^{n+1}+M_{j}^{n}}{2}\right)\left\langle\frac{\partial \phi_{j}}{\partial y}, \phi_{i}\right\rangle=N_{4}-\beta_{x} \sum_{j}\left(\frac{I_{j}^{n+1}+I_{j}^{n}}{2}\right) \sum_{k}\left(\frac{M_{k}^{n+1}+M_{k}^{n}}{2}\right)\left\langle\phi_{j} \phi_{k}, \phi_{i}\right\rangle \\
& -\xi \sum_{j}\left(\frac{M_{j}^{n+1}+M_{j}^{n}}{2}\right)\left\langle\phi_{j}, \phi_{i}\right\rangle \text {. } \\
& \sum_{j}\left(\frac{P_{j}^{n+1}-P_{j}^{n}}{\Delta t}\right)\left\langle\phi_{j}, \phi_{i}\right\rangle+\alpha_{p} \sum_{j}\left(\frac{P_{j}^{n+1}+P_{j}^{n}}{2}\right)\left\langle\nabla \phi_{j} \| \nabla \phi_{i}\right\rangle+\beta_{p_{1}} \sum_{j}\left(\frac{P_{j}^{n+1}+P_{j}^{n}}{2}\right)\left\langle\frac{\partial \phi_{j}}{\partial x}, \phi_{i}\right\rangle \\
& +\beta_{p_{2}} \sum_{j}\left(\frac{P_{j}^{n+1}+P_{j}^{n}}{2}\right)\left\langle\frac{\partial \phi_{j}}{\partial y}, \phi_{i}\right\rangle=\beta_{x} \sum_{j}\left(\frac{I_{j}^{n+1}+I_{j}^{n}}{2}\right) \sum_{k}\left(\frac{M_{k}^{n+1}+M_{k}^{n}}{2}\right)\left\langle\phi_{j} \phi_{k}, \phi_{i}\right\rangle \\
& -\xi \sum_{j}\left(\frac{P_{j}^{n+1}+P_{j}^{n}}{2}\right)\left\langle\phi_{j}, \phi_{i}\right\rangle \text {. }
\end{aligned}
$$

The matrix formulation for the iterative process is:

$$
\begin{aligned}
& C_{s}\left(P^{n+1}, P^{n}\right) S^{n+1}=D_{s}\left(P^{n+1}, P^{n}\right) S^{n}, \\
& C_{I}(c) I^{n+1}=D_{I}\left(S^{n}, S^{n+1}, P^{n}, P^{n+1}\right) I^{n}, \\
& C_{R}(c) R^{n+1}=D_{R}\left(I^{n}, I^{n+1}\right) R^{n}, \\
& C_{M}\left(I^{n}, I^{n+1}\right) M^{n+1}=D_{M}\left(I^{n}, I^{n+1}\right) M^{n}, \\
& C_{P}(c) P^{n+1}=D_{P}\left(I^{n}, I^{n+1}, M^{n}, M^{n+1}\right) P^{n} .
\end{aligned}
$$

Where $C_{I}(c), C_{R}(c)$ and $C_{P}(c)$ are matrices of constant coefficients.

Solution algorithm proposal:

To solve the system we used a predictor-corrector method, [7, 8, 32].

We start with the initial conditions $\left(S^{0}, I^{0}, R^{0}, M^{0}, P^{0}\right)$, and we look for the 
first approximation $\left(S^{1}, I^{1}, R^{1}, M^{1}, P^{1}\right)$.

For $S^{*}$ we solve: $\quad C_{s}\left(P^{0}, P^{0}\right) S^{*}=D_{s}\left(P^{0}, P^{0}\right) S^{0}$.

Then look for $I^{*}, \quad C_{I}(c) I^{*}=D_{I}\left(S^{0}, S^{*}, P^{0}, P^{0}\right) I^{0}$.

We calculate $R^{*}, \quad C_{R}(c) R^{*}=D_{R}\left(I^{0}, I^{*}\right) R^{0}$.

We calculate $M^{*}, \quad C_{M}\left(I^{0}, I^{*}\right) M^{*}=D_{M}\left(I^{0}, I^{*}\right) M^{0}$.

We calculate $P^{*}, \quad C_{P}(c) P^{*}=D_{P}\left(I^{0}, I^{*}, M^{0}, M^{*}\right) P^{0}$.

We obtained $\left(S^{*}, I^{*}, R^{*}, M^{*}, P^{*}\right)$.

Now, with $\left(S^{0}, I^{0}, R^{0}, M^{0}, P^{0}\right)$ and $\left(S^{*}, I^{*}, R^{*}, M^{*}, P^{*}\right)$ we look for $\left(S^{* *}, I^{* *}, R^{* *}, M^{* *}, P^{* *}\right)$, analogously to the previous scheme.

We calculate $S^{* *}$ solving: $\quad C_{s}\left(P^{0}, P^{*}\right) S^{* *}=D_{s}\left(P^{0}, P^{*}\right) S^{0}$.

We calculate $I^{* *}, \quad C_{I}(c) I^{* *}=D_{I}\left(S^{0}, S^{* *}, P^{0}, P^{*}\right) I^{0}$.

We calculate $R^{* *}, \quad C_{R}(c) R^{* *}=D_{R}\left(I^{0}, I^{* *}\right) R^{0}$.

We calculate $M^{* *}, \quad C_{M}\left(I^{0}, I^{*}\right) M^{* *}=D_{M}\left(I^{0}, I^{*}\right) M^{0}$.

We calculate $P^{* *}, \quad C_{P}(c) P^{* *}=D_{P}\left(I^{0}, I^{* *}, M^{0}, M^{* *}\right) P^{0}$.

We declare the conditions of convergence and we continue the process. Then, for $p \in \mathbb{N}:\left(S^{1}=S^{p *}, I^{1}=I^{p *}, R^{1}=R^{p *}, M^{1}=M^{p *}, P^{1}=P^{p *}\right)$. Repeat the process for $n$ y $\left(S^{n}, I^{n}, R^{n}, M^{n}, P^{n}\right)$ is the approximate solution of the model. 


\section{5}

\section{Graphical User Interface}

\section{1}

\section{Graphical User Interface}

The graphical user interface allows you to obtain the graphical solution of the model 3.1 presented in chapter 3, section 3.1. Was made in Matlab the interface presented, this allows users to use the model more quickly and efficiently without a deep knowledge of mathematical theory. The parameters, variables and initial conditions are maintained.

Validation of input data related to model conditions:

- The entry of the parameters are numbers and between zero and one.

- The entry of the initial conditions are numbers and greater than or equal to zero.

- The time is number and greater than zero.

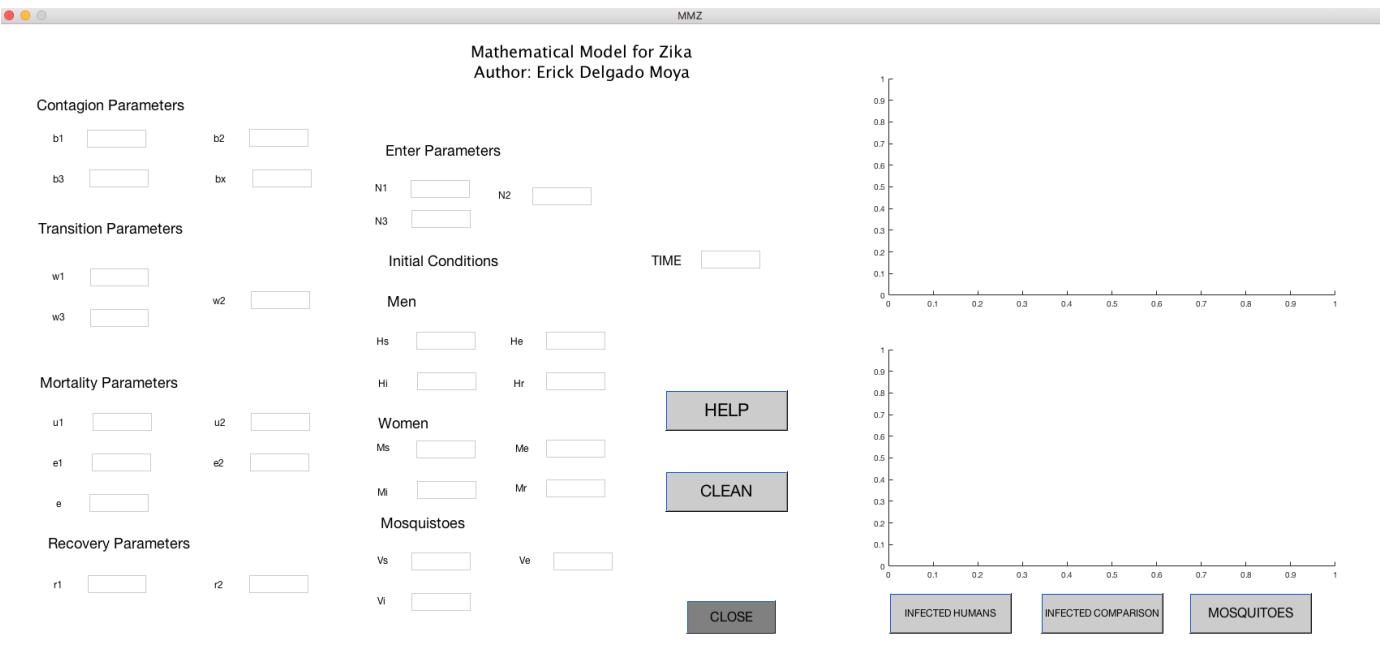

Figure 5.1.1: Graphical User Interface.

Function of buttons:

- Help: Gives a general explanation about parameter input, initial conditions, and output interface. 
- Clean: Clean the entries and graphics for a new simulation.

- Infected Humans: Graphical trend for the infected population by gender.

- Comparison: Graphical comparison for infected and recovered populations by gender.

- Mosquitoes: Graphical trend of susceptible and infected mosquitoes.

The interface has included a document that has all the considerations for the use of it.

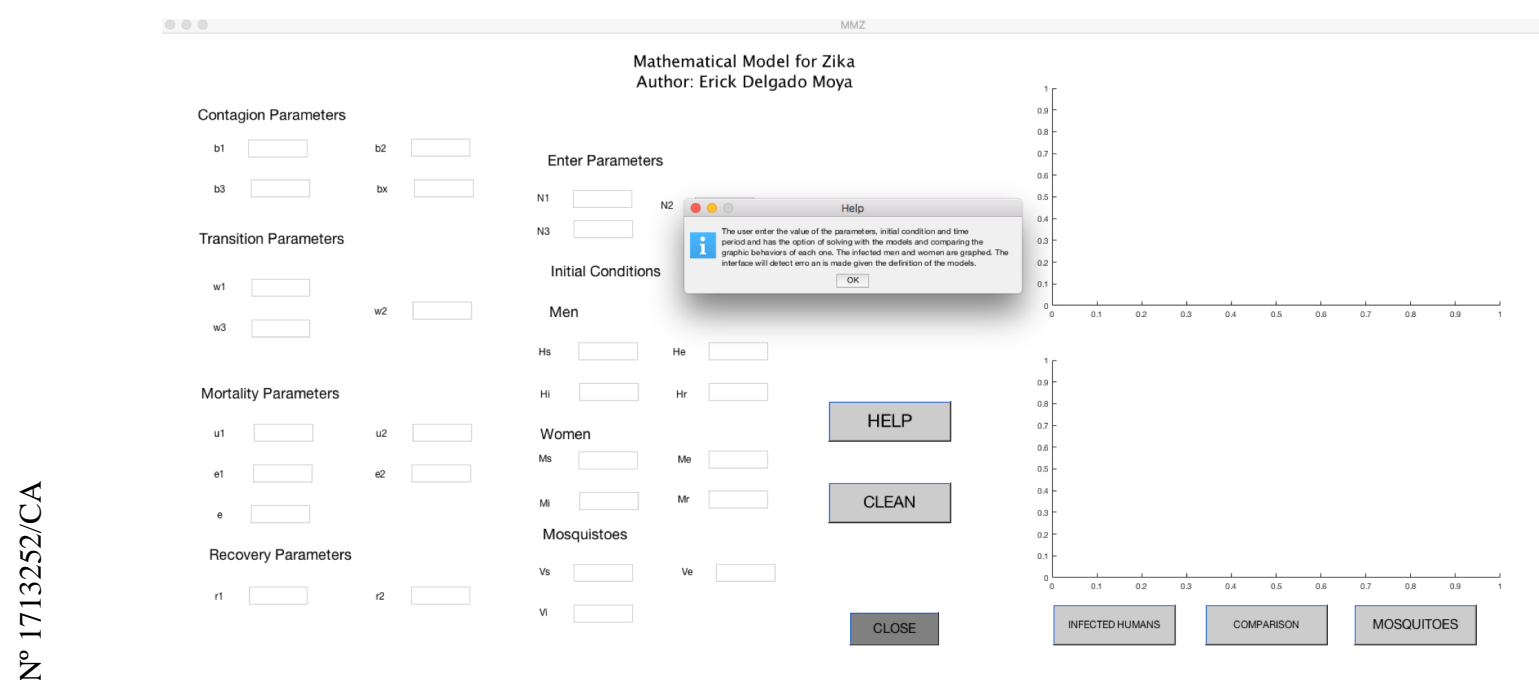

Figure 5.1.2: Graphical User Interface, button Help.

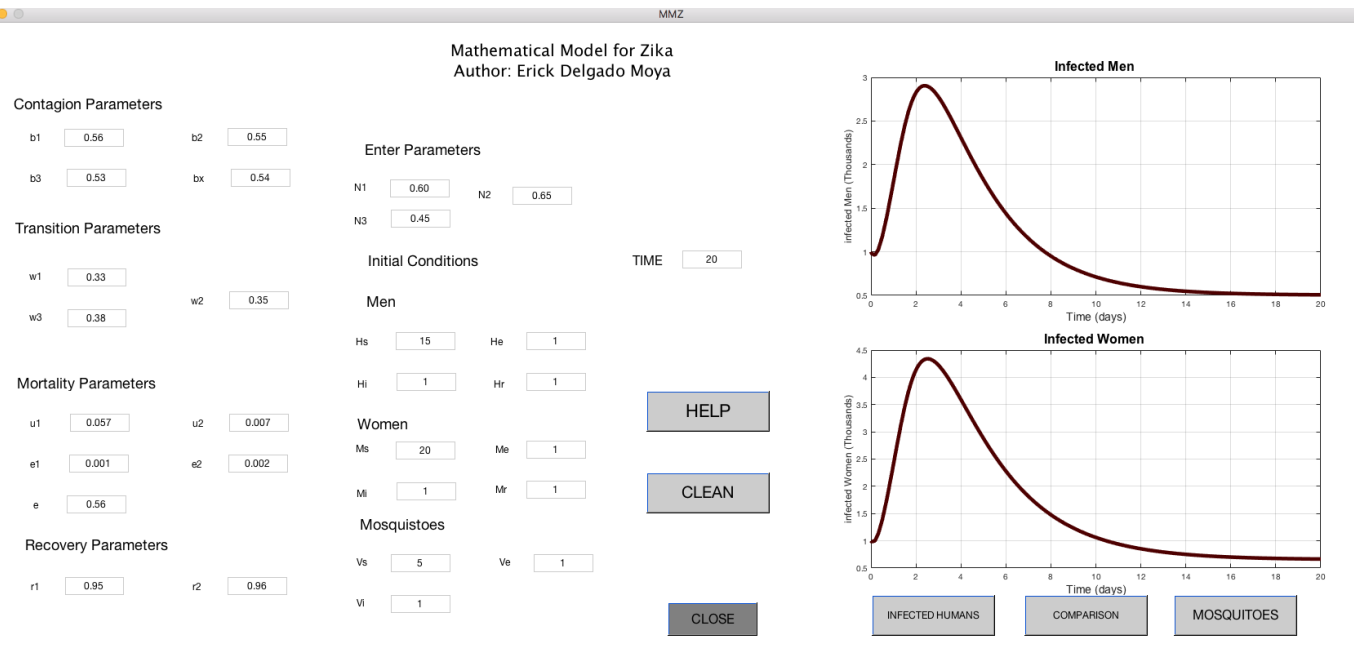

Figure 5.1.3: Graphical User Interface, button Infected Humans. 


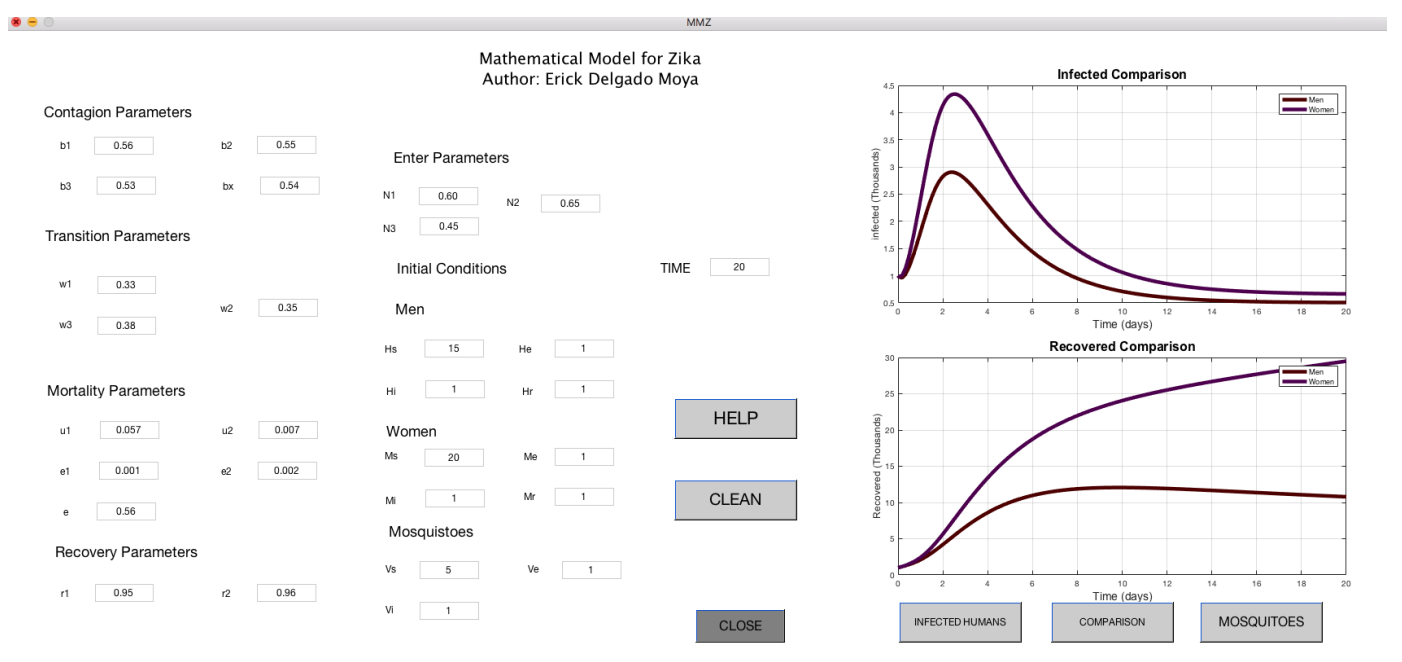

Figure 5.1.4: Graphical User Interface, button Comparison.

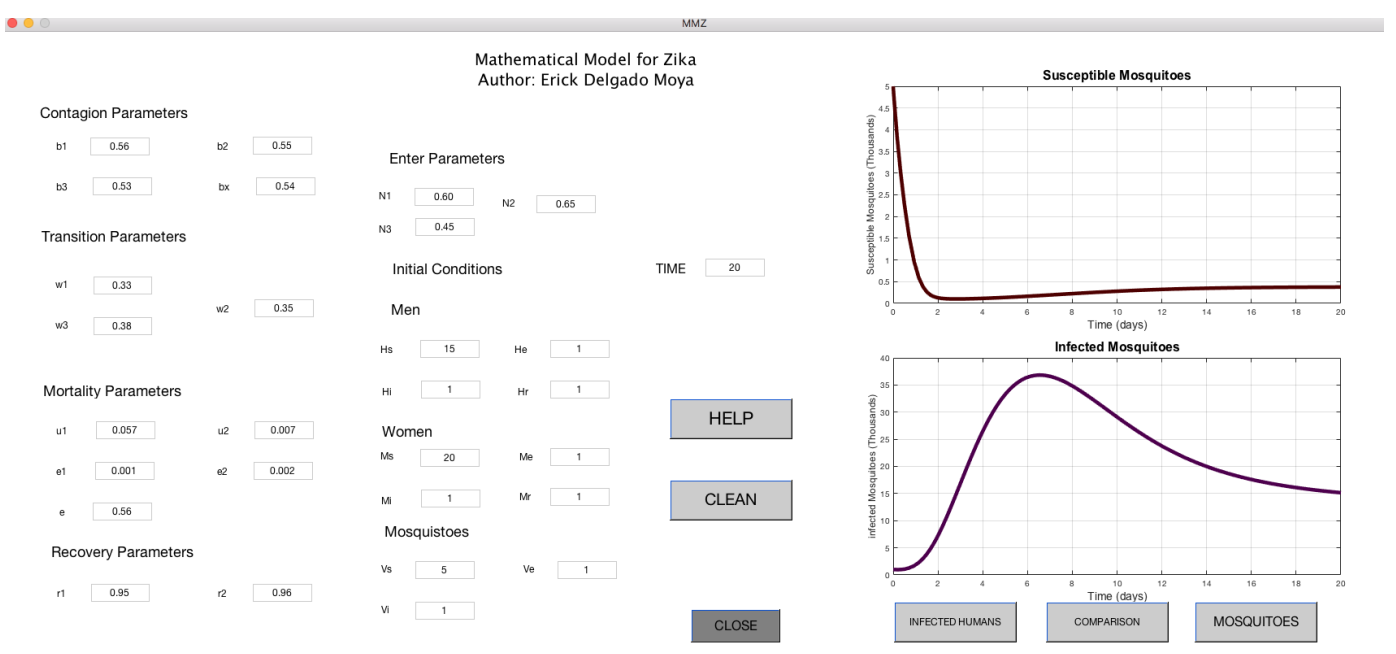

Figure 5.1.5: Graphical User Interface, button Mosquitoes. 


\section{6}

\section{Appendix}

Lemma 6.1. (The Grönwall Inequality) Let $z(t)$ be a non-negative continuous function on $\left[t_{0}, t_{1}\right]$ where $t_{0}<t_{1}$. Assume that there are constants $C, L \geq 0$ such that

$$
z(t) \leq C+L \int_{t_{0}}^{t} z(s) d s
$$

for all $t \in\left[t_{0}, t_{1}\right]$. Then

$$
z(t) \leq C \exp \left(L\left(t-t_{0}\right)\right)
$$

for all $t \in\left[t_{0}, t\right]$.

Proof. We can assume that $C$ is strictly positive. Indeed, if (6.1) holds with $C=0$ then it holds with any $C>0$. Therefore, (6.2) holds with any $C>0$, whence it follows that it holds with $C=0$. Hence, assume in the sequel that $C>0$. This implies that the right hand side of (6.1) is positive. Set

$$
F(t)=C+L \int_{t_{0}}^{t} z(s) d s
$$

and observe that $F$ is differentiable and $F^{\prime}=L z$. It follows (6.1) that $z \leq F$ whence $F^{\prime}=L z \leq L F$.

This is a differential inequality for $F$ that can be solved similarly to the separable ODE. Since $F>0$, dividing by $F$ we obtain

$$
\frac{F^{\prime}}{F} \leq L
$$

whence by integration

$$
\ln \frac{F(t)}{F\left(t_{0}\right)}=\int_{t_{0}}^{t} \frac{F^{\prime}(s)}{F(s)} \leq \int_{t_{0}}^{t} L d s=L\left(t-t_{0}\right),
$$

for all $t \in\left[t_{0}, t_{1}\right]$. It follows that

$$
F(t) \leq F\left(t_{0}\right) \exp \left(L\left(t-t_{0}\right)\right)=C \exp \left(L\left(t-t_{0}\right)\right)
$$

Using again (6.1), that is, $z \leq F$, we obtain (6.2). 
Lemma 6.2. Let $H$ be a non-singular $M$-matrix and suppose $B$ and $B H^{1}$ have the $Z$ sign pattern, Then $B$ is a non-singular $M$-matrix if and only if $B H^{-1}$ is a non- singular matrix.

The proof is in [14].

Proof. The forward implication is stated in a slightly different form [12] and the reverse implication is stated in [4].

In general, this lemma does not hold if $B$ a singular $M$-matrix. It can be shown to hold if $B$ is singular and irreducible.

Lemma 6.3. Let $H$ be a non-singular $M$-matrix and suppose $K \geq 0$. Then,

i. $(H-K)$ is non-singular $M$-matrix if and only if $(H-K) H^{-1}$ is a non-singular $M$-matrix.

ii. $(H-K)$ is singular $M$-matrix if and only if $(H-K) H^{-1}$ is a singular $M-$ matrix.

Proof. Let $B=H-K$. Then both $B$ and $B H^{-1}=I-K H^{-1}$ have the $Z$ sign pattern. (Recall that $H^{-1} \geq 0$ since $H$ is a non-singular $M$-matrix). Hence, Lemma 6.2 statement (i.). A separate continuity argument can be constructed for each implication in the singular case. 


\section{Bibliography}

[1] S. ALIZON AND C. MAGNUS. Modelling the course of an hiv infection: Insights from ecology and evolution. Viruses, 2:19842013, 2013.

[2] V. H. BADSHAH, P. PORWAL AND V. TIWARI. Mathematical modelling and role of dynamics in epidemiology. International Journal of Computational Science and Mathematics, 5(1):4-10, 2013.

[3] C. T. H. BAKER, C. A. H. PAUL AND D. R. WILLÉ. Issues in the numerical solution of evolutionary delay differential equations. Adv. Comp. Math., 3:171-196, 1995.

[4] T. BERGE, J. M. S. LUBUMA, G. M. MOREMEDI, N. MORRIS AND R. KONDERA- SHAVA. A simple mathematical model for ebola in africa. Journal of Biological Dynamics, 11(1):42-74, 2017.

[5] A. BERMAN, R.J. PLEMMONS. Nonnegative Matrices in the Mathematical Sciences. Academic Press, 1970.

[6] E. BONYAH AND K. C. OKOSUN. Mathematical modeling of zika virus. Asian Pacific Journal of Tropical Disease, 6 (9):673-679, 2016.

[7] J. C. BUTCHER. Numerical method for ordinary differential equations. John Wiley and Sons, 2003.

[8] C. CHICONE. Ordinary differential equation with application. Springer Science and Business Media, 1999.

[9] P. G. CIARLET. The finite element method for elliptic problem. SIAM, 2002.

[10] J. CRANK AND E. NICOLSON. A practical method for numerical evaluation of partial differential equations of the heat conduction type. Proc. Camb. Phil. Soc., 43(1):50-67, 1947.

[11] O. S. DEEPA, S. NALLAMALLI, L. N. S. NAIKAND G. V. S. TEJA. Mathematical model for transmission of ebola. Procedia Computer Science, 48:741-745, 2015. 
[12] R. A. HORN, C.R. JONHSON. Topic in Matrix Analysis. Cambridge University, 1991.

[13] W. R. DERRICK AND S. I. GROSSMAN . Elementary Differential Equations with Applications. Addison-Wsley Pub. Co., 1976.

[14] P. V. DRIESSCHE AND J. WATMOUGH. Reproduction numbers and sub-threshold endemic equilibria for compartmental models of disease transmission. Mathematical Biosciences, 180:29-48, 2002.

[15] G. W. A. DICK, S. F. KITCHEN AND A. J. HADDOW. Zika virus i. isolations and serogical specifity. Trans R Soc Trop Med Hyg, 46(5):509-520, 1952.

[16] G. W. A. DICK, S. F. KITCHEN AND A. J. HADDOW. Zika virus ii. pathogenicity and physical properties. Trans R Soc Trop Med Hyg, 46:521-534, 1952.

[17] O. DiekMAM, J. A. P. HEESTERBEEK AND M. G. ROBERTS. The construction of next-generation matrices for compartimental epidemical models. J. Royal Society Interface, 7:873-885, 2010.

[18] R. D. DRIVER. Ordinary and delay differential equations. New York: Springer Verlag, 1977.

[19] L. ESTEVA AND C. VARGAS. Analysis of a dengue disease transmission model. Mathematical Biosciences, 150:131-151, 1998.

[20] T. G. KASSEN AND E. J. D. GARBA. A mathematical model for the spatial spread oh hiv in heterogeneous population. Mathematical Theory and Modeling, 6(4):95-104, 2016.

[21] M. J. KEELING AND L. DANON. Mathematical modelling of infectious diseases. Brithsh Medical Bulletin, 92(1):33-42, 2014.

[22] C. E. KNIGHT. The finite element method in mechanical design. PWS-KENT Publishing Company, 1993.

[23] H. LIN AND F. WANG. On a reaction-diffusion system modeling the dengue transmission with nonlocal infections and crowding effects. Applied Mathematics and Computation, 248:184-194, 2014.

[24] E. M. LOTFI, M. MAZIANE, K. HATTAF AND NOURA YOUSTI. Partial differential equations of an epidemic model with spatial difussion. International Journal of Partial Differential Equations, 2014. 
[25] M. M. MACUFA, J. F. MAYER AND A. KRINDGES. Difussion of malaria in mozambique. modeling with computational simulations. Biomatemática, 25:161-184, 2015.

[26] N. A. MAIDANA AND H. M. YANG. A spatial model to describe the dengue propagation. TEMA, Tend. Mat. Apli. Comp., 8(1):83-92, 2007.

[27] R. MATTHEIJ AND J. MOLENAR. Ordinary differential equations in theory and practice. Published by Society for Industrial and Applied Mathematic, 2002.

[28] T. O. OLUYO AND M. O. ADEYEMI. Mathematical analysis of zika epidemic model. IOSR-JM, 12 (6):21-33, 2002.

[29] R. PENG AND X. Q. ZHAO. A reaction-diffusion sis epidemic model in a time periodic environment. Nonlinearity, 25:1451- 1471, 2012.

[30] C. POZRIKIDIS. Introduction to Theoretical and Computational Fluid Dynamics. Oxford University Press, 1997.

[31] X. REN, Y. TIAN, L. LIU AND X. LIU. A reaction difussion withen-host hiv model with cell-to-cell transmision. J. Math. Biol., 76:1831-1872, 2018.

[32] G.W. RECKTENWALD. Finite-difference aproximations to the heat equation.

[33] D. P. SHUTT, C. A MANORE, S. PANKAVICH, A. T. PORTER AND S. Y DEL VALLE. Estimating the reproductive number, total outbreak size, and reporting rates for zika epidemics in south and central america. Epidemics, 21:63-79, 2017.

[34] I. M. SMITH. Programming the Finite Element Method. John Wiley Sons, Chichester, 1998.

[35] A. K. SUPRIATNA, E. SOEWONO AND S. VANGILS. A two-ages classes of dengue transmission model. Math. Biosc., 216:114-121, 2008.

[36] T. T. TIEMI, N. A. MAIDAN, W. C. FERREIRA JR, P. PAULINO AND H. YANG. Mathematical models for the aedes aegypti dispersal dynamics: Travelling waves by wing and wind. Bulletin of Mathematical Biology, 67:509-528, 2005.

[37] A. N.TIJONOV, AND A. A. SAMARSKY. Ecuaciones de la Fisica Matemática. Editorial Mir, 1980. 
[38] J. W. THOMAS. Numerical Partial Differential Equations: Finite Difference Methods. Springer Science LLC.

[39] D. WORDARZ AND M. A. NOWAK. Mathematical model of hiv pathogenesis and treatment. Bio ESSAYS, 24:1178-1187, 2014. 